\title{
Output-Feedback Control of a Chaotic MEMS Resonator for Oscillation Amplitude Enhancement
}

\author{
Alexander Jimenez-Triana, ${ }^{1}$ Guchuan Zhu, ${ }^{2}$ and Lahcen Saydy ${ }^{2}$ \\ ${ }^{1}$ Department of Control Engineering, Universidad Distrital Francisco José de Caldas, Calle 74 Sur No. 68A-20, Bogotá, Colombia \\ ${ }^{2}$ Department of Electrical Engineering, École Polytechnique de Montréal, Montréal, QC, Canada H3C 3A7 \\ Correspondence should be addressed to Alexander Jimenez-Triana; alex_jimenez_triana@yahoo.es
}

Received 12 May 2014; Accepted 6 August 2014; Published 9 September 2014

Academic Editor: Ben T. Nohara

Copyright (c) 2014 Alexander Jimenez-Triana et al. This is an open access article distributed under the Creative Commons Attribution License, which permits unrestricted use, distribution, and reproduction in any medium, provided the original work is properly cited.

\begin{abstract}
The present work addresses the problem of chaos control in an electrostatic MEMS resonator by using an output-feedback control scheme. One of the unstable orbits immersed in the chaotic attractor is stabilized in order to produce a sustained oscillation of the movable plate composing the microstructure. The orbit is carefully chosen so as to produce a high amplitude oscillation. This approach allows the enhancement of oscillation amplitude of the resonator at a reduced control effort, since the unstable orbit already exists in the system and it is not necessary to spend energy to create it. Realistic operational conditions of the MEMS are considered including parametric uncertainties in the model and constraints due to the difficulty in measuring the speed of the plates of the microstructure. A control law is constructed recursively by using the technique of backstepping. Finally, numerical simulations are carried out to confirm the validity of the developed control scheme and to demonstrate the effect of controlling orbits immersed in the chaotic attractor.
\end{abstract}

\section{Introduction}

This paper addresses the problem of chaos control in an electrostatic MEMS resonator with parametric uncertainties and constraints in the measure of its state. The considered device is a structure with dual drive using differential actuation, as shown in Figure 1.

Many MEMS devices exhibit chaotic behaviour in certain operational conditions [1-4]. It is observed in recent results concerning the improvement of the output energy in oscillators using MEMS [5] that controlling MEMS in chaotic mode can be useful to increase the amplitude of the periodic oscillations of these systems. Therefore, controlling chaos in MEMS can enhance the performance of different systems in which an accurate control of amplitude of oscillation is mandatory.

Chaos control has been extensively studied in the literature. Different methods have been applied to stabilize equilibrium points and unstable orbits immersed in the chaotic attractor or to track orbits that can be chaotic. The considered approaches cover the application of a wide range of control techniques, from the use of Lyapunov theory to stabilize chaotic oscillators designed via local linearization or linear feedback [6, 7], to optimal control [8], robust control [9], and adaptive control [10], to name a few. Chaos control in MEMS is reported recently in $[1,11]$. In [1] a fuzzy control algorithm is applied to control the chaotic motion and track a sinusoidal orbit. In [11] an adaptive control is used to control an uncertain model of a MEMS device and to synchronize it with a class of uncertain chaotic oscillators. Nevertheless, the enhancement of oscillation amplitude for MEMS resonators by appropriate control techniques remains an interesting and open research subject.

The oscillation amplitude of electrostatic MEMS resonators is restricted by the so-called pull-in phenomena related to a bifurcation arising in electrostatic actuation [12]. The stable traveling range is limited to one-third of its full gap due to the instability beyond that point. The pull-in phenomena can be used to accelerate the reaction speed for switching structures. However, it limits the stable operational range of the device, which is mandatory for applications requiring a sustained oscillation of the plates. 


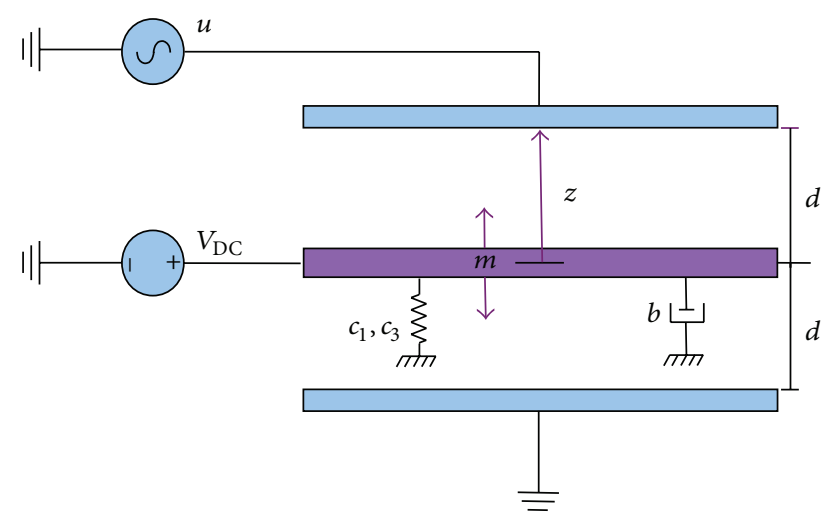

FIGURE 1: Schematic of the electrostatically actuated MEMS resonator.

In order to operate electrostatic MEMS devices beyond the pull-in, one can use closed-loop control schemes (see, e.g., $[13,14])$. However, it may require a considerable control effort to produce an arbitrary trajectory. In order to increase the amplitude of periodic oscillations of the device with a low energy consumption, we consider in the present work controlling the resonator in chaotic mode to stabilize an orbit which is part of the chaotic attractor.

There are different ways to select this orbit. One can pick an unstable periodic orbit embedded in the chaotic attractor, take an orbit close to an unstable periodic one, or even choose a part of a chaotic trajectory that has an almost periodic behavior.

In the present work, we tackle the amplitude enhancement problem by stabilizing one of these orbits to produce a high amplitude oscillation.

There are some constraints that must be considered in a practical implementation of a chaos control scheme applied to MEMS. In particular, it is very difficult to sense the velocity of the plates during the operation of the device, which motivates the use of output-feedback control techniques. In this work, the output is chosen as the position of the plates, and, in order to implement the control, the velocity must be estimated by constructing an observer. We deal with the uncertainties in the model and the constraints on sensing the velocity by designing a controller by means of an adaptive observer backstepping scheme.

The organization of the rest of the paper is as follows. In Section 2, the dynamics of a chaotic MEMS actuator are briefly explained. Then, the design of an adaptive observer control scheme is presented in Section 3. Simulations are carried out and the results are presented in Section 4. Finally, concluding remarks are given in Section 5.

\section{Dynamics of a Chaotic MEMS Resonator}

2.1. Model of the Chaotic MEMS. The MEMS device considered in this study is presented in Figure 1, which is based on a model given in $[1,15]$. This device is modeled by a nonlinear mass-spring-damper system with external electrostatic actuation, expressed as

$$
m \ddot{z}+b \dot{z}+c_{1} z+c_{3} z^{3}=F,
$$

where $m$ is the mass of the movable structure, $b$ is the damping constant, $c_{1}$ and $c_{3}$ are the linear and cubic stiffness coefficients, respectively, and $F$ is the electrostatic actuation force given by

$$
F=\frac{1}{2} \frac{C_{0} d}{(d-z)^{2}}\left(V_{\mathrm{DC}}+u\right)^{2}-\frac{1}{2} \frac{C_{0} d}{(d+z)^{2}} V_{\mathrm{DC}}^{2}
$$

In (2), $C_{0}$ is the capacitance of the actuator at rest, which has an initial gap $d$ between the movable and fixed plates. The input voltage $V_{i}=\left(V_{\mathrm{DC}}+u\right)$ is applied as shown in Figure 1 and contains two components: a DC voltage $V_{\mathrm{DC}}$ and a forcing voltage $u$, which may be a control signal that varies according to control objectives.

Note that, in the present work, we ignore the dynamics of the driving circuit by assuming that they are much faster than those of the mechanical structure. Moreover, as the MEMS are used as a resonator, we assume that the system operates in such a way that the moveable and fixed plates do not come into contact. Hence, we do not take into account contact dynamics. Nevertheless, for those applications, where the contact may occur, a different model should be considered to capture the dynamics of impacts and the control strategy should be modified adequately [16].

In order to facilitate control system design and analysis, it is convenient to work in normalized coordinates and redefine the time variable. For this purpose, we define the new variables $\psi=z / d, \tau=\omega_{0} t$, and $\widetilde{u}=u A$ and introduce the following dimensionless constants:

$$
\begin{gathered}
\sigma=\frac{c_{1}}{m \omega_{0}^{2}}, \quad \eta=\frac{c_{3} d^{2}}{m \omega_{0}^{2}}, \quad \delta=\frac{C_{0} V_{\mathrm{DC}}^{2}}{2 m \omega_{0}^{2} d^{2}}, \\
A=2 \delta \frac{1}{V_{\mathrm{DC}}}, \quad \mu=\frac{b}{m \omega_{0}},
\end{gathered}
$$

where $\omega_{0}$ is the natural frequency defined by

$$
\omega_{0}=\sqrt{\frac{c_{1}}{m}} .
$$

In this way, by assuming that the DC voltage is sufficiently higher than the amplitude of the forcing voltage $u$, the dimensionless equation of motion (1) may be fairly approximated by

$$
\begin{aligned}
\ddot{\psi}+\mu \dot{\psi}+\sigma \psi+\eta \psi^{3} \\
=\delta\left(\frac{1}{(1-\psi)^{2}}-\frac{1}{(1+\psi)^{2}}\right)+\frac{\widetilde{u}}{(1-\psi)^{2}} .
\end{aligned}
$$

It can be shown that system (5) behaves chaotically by selecting $u=V_{\mathrm{AC}} \sin (\widetilde{\omega} t)$ and $V_{\mathrm{AC}} A=0.04, \widetilde{\omega}=0.5$, $\delta=0.338, \mu=0.01, \sigma=1$, and $\eta=12$, as indicated in the Lyapunov exponents diagram in Figure 2. The Lyapunov exponents are calculated by using the algorithm proposed 


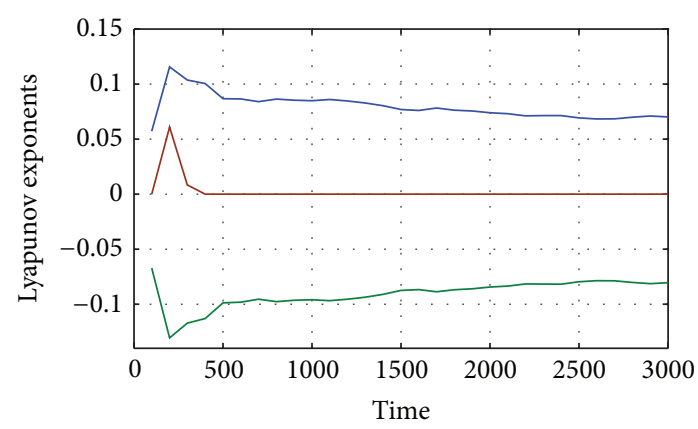

FIGURE 2: Lyapunov exponents for the system (5) with the set of parameters: $\delta=0.338, A=0.04, \omega=0.5, \sigma=1, \mu=0.01$, and $\eta=12$.

in [17]. The existence of one positive Lyapunov exponent confirms the sensitive dependence on initial conditions of the system.

System (5) can be written in state space form as

$$
\begin{gathered}
\dot{\psi}_{1}=\psi_{2}, \\
\dot{\psi}_{2}=-\mu \dot{\psi}_{2}-\sigma \psi_{1}-\eta \psi_{1}^{3}+f\left(\psi_{1}\right)+\frac{\widetilde{u}}{\left(1-\psi_{1}\right)^{2}}, \\
y=\psi_{1},
\end{gathered}
$$

where

$$
f\left(\psi_{1}\right)=\delta\left(\frac{1}{\left(1-\psi_{1}\right)^{2}}-\frac{1}{\left(1+\psi_{1}\right)^{2}}\right)
$$

and $y$ is the output.

2.2. Unstable Orbits in the Strange Attractor. The main idea to obtain a sustained oscillation of the MEMS with a low energy consumption is not to create a new orbit different from those already present in the attractor. The orbit could be an unstable periodic orbit embedded in the chaotic attractor, an orbit close to an unstable periodic one, or even part of a chaotic trajectory which has an almost periodic behavior.

In order to find one of such orbits, one may measure the phase space dynamics of the system and obtain a set of points from which one can extract the orbit by applying the close return method [18]. Note that other approaches, such as the shooting method [19], can also be used for this purpose. With the aim of simplifying the discussion, we will only consider systems, where the state $x=\left(x_{1}, x_{2}\right) \in \mathbb{R}^{2}$ and an external periodic force is applied. Under these conditions, a Poincaré map can be easily constructed from stroboscopically logging the value of the variable $x$ at multiples of the period $T$ of the forcing. Time can be seen as one of the states, and the problem of finding periodic orbits reduces to find fixed points in $\mathbb{R}^{2}$ on the Poincaré surface.

The Poincaré map may be defined as follows. Given the differential equation

$$
\dot{x}=f(x, t), \quad x \in \mathbb{R}^{2},
$$

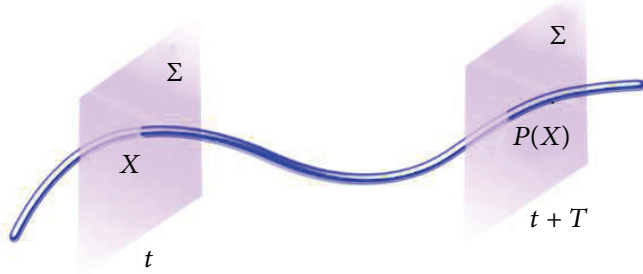

Figure 3: Poincaré section $\Sigma$ and Poincaré map $P$ for the periodic vector field defined by (8) and (9).

with periodic phase space of period $T$ such that

$$
f(x, t)=f(x, t+T),
$$

then the Poincaré map is defined as

$$
\begin{gathered}
P: \Sigma \longrightarrow \Sigma, \\
x \longmapsto \varphi(T, x),
\end{gathered}
$$

where $\varphi(t, \cdot)$ denotes the flow generated by (8) and $\Sigma$ is a Poincaré section as shown in Figure 3.

The procedure for finding orbits with almost periodic behaviour is similar to the one of finding periodic orbits. In both cases, the image under the Poincaré map of a point close to the orbit, which intersects the Poincare surface, is close to the point. Finding such point is the aim of the close return method.

For the purpose of applying the method, one records discretely sampled time series $x(0), x\left(T_{s}\right), \ldots, x\left((N-1) T_{s}\right)$ containing $N$ points, where $T_{s}$ is the sample time. Then, for a $k$ given, one takes a point $x\left(k T_{s}\right)$ and its iteration $m$ on the map $x\left(k T_{s}+m T\right)$ and evaluates the distance $d\left(x\left(k T_{s}\right), x\left(k T_{s}+m T\right)\right)$ between the points, by using, for example, the Euclidean norm. If the distance is less than a small $\epsilon_{s}$, then this is a $m$ period orbit that could be used.

In order to show the application of the close return method, we use three different time series from which we extract unstable orbits of different periods. Each of these series comprises a set of $N=120000$ points with sample time of $T_{s}=0.01$, which corresponds to a logging time of approximately 100 times the period of the forcing term. The orbits were obtained by using $\epsilon_{s}=0.005$ and can be seen in Figures 4,5 , and 6 . The figures suggest that the bifurcations occurred when the period increases.

In order to enhance the oscillation amplitude of the plates, we chose an unstable orbit surrounding the chaotic attractor, which produces a high amplitude periodic output. This orbit, shown in Figure 7, was used as the reference trajectory in the simulation given subsequently. It can be approximated by its truncated Fourier series up to five terms, given by

$$
\begin{aligned}
y_{r}= & a_{0}+a_{1} \cos \left(w_{1} t\right)+b_{1} \sin \left(w_{1} t\right)+a_{2} \cos \left(2 w_{1} t\right) \\
& +b_{2} \sin \left(2 w_{1} t\right)+a_{3} \cos \left(3 w_{1} t\right)+b_{3} \sin \left(3 w_{1} t\right) \\
& +a_{4} \cos \left(4 w_{1} t\right)+b_{4} \sin \left(4 w_{1} t\right)+a_{5} \cos \left(5 w_{1} t\right) \\
& +b_{5} \sin \left(5 w_{1} t\right),
\end{aligned}
$$




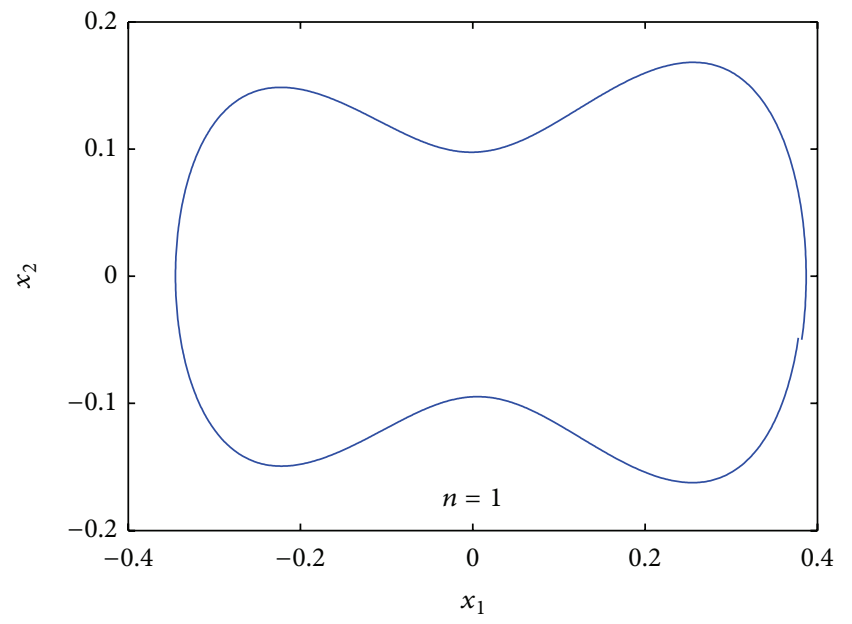

(a)

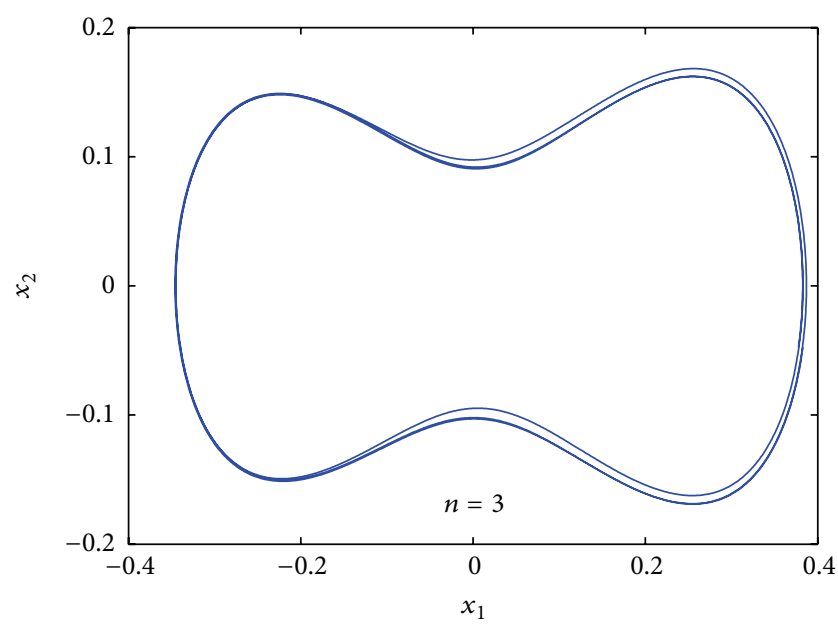

(c)

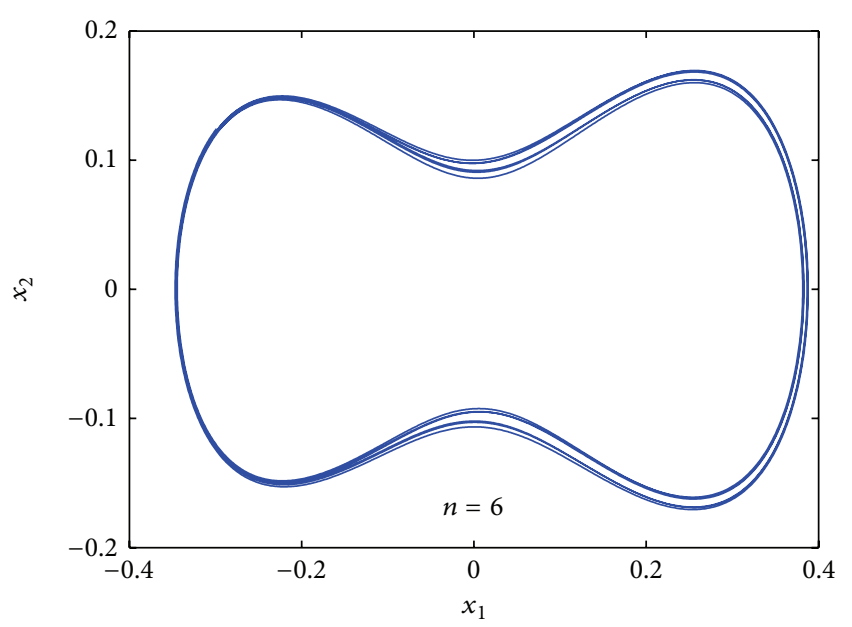

(e)

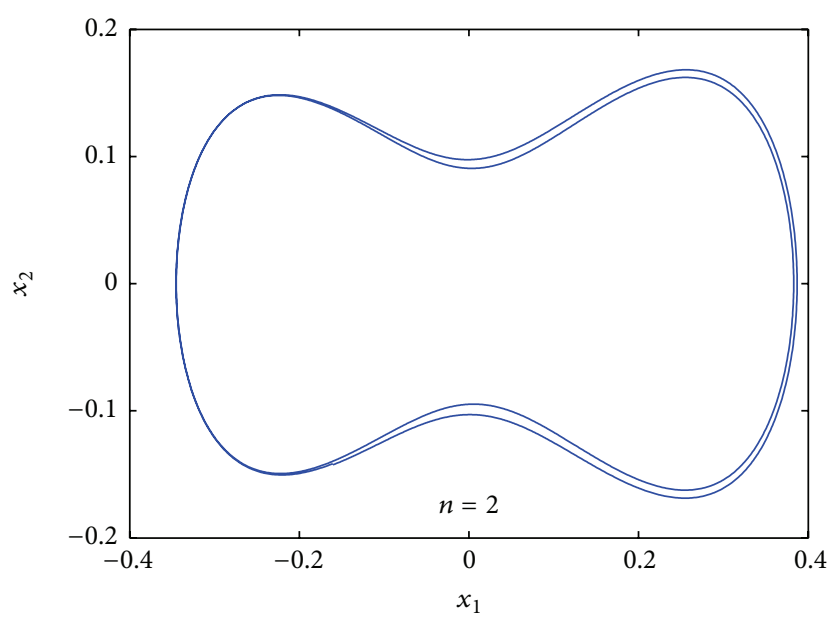

(b)

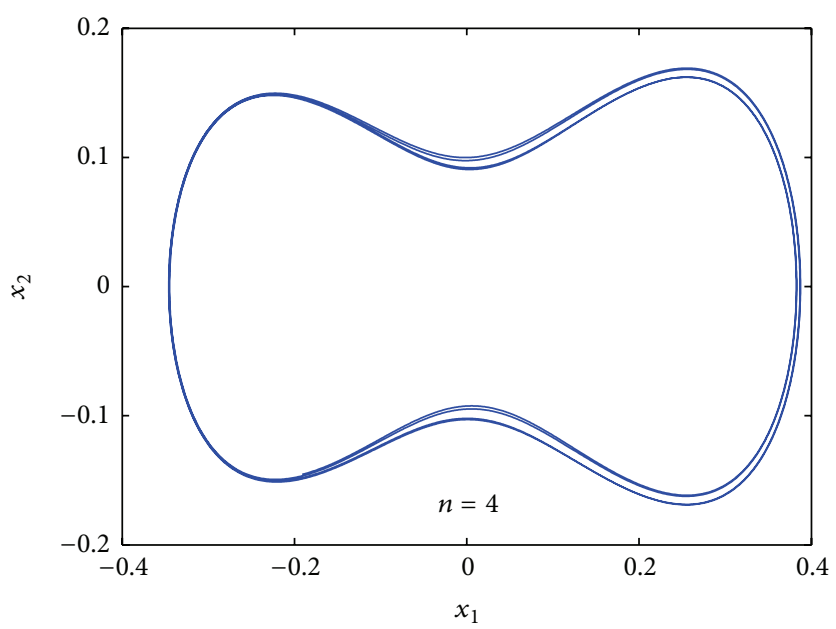

(d)

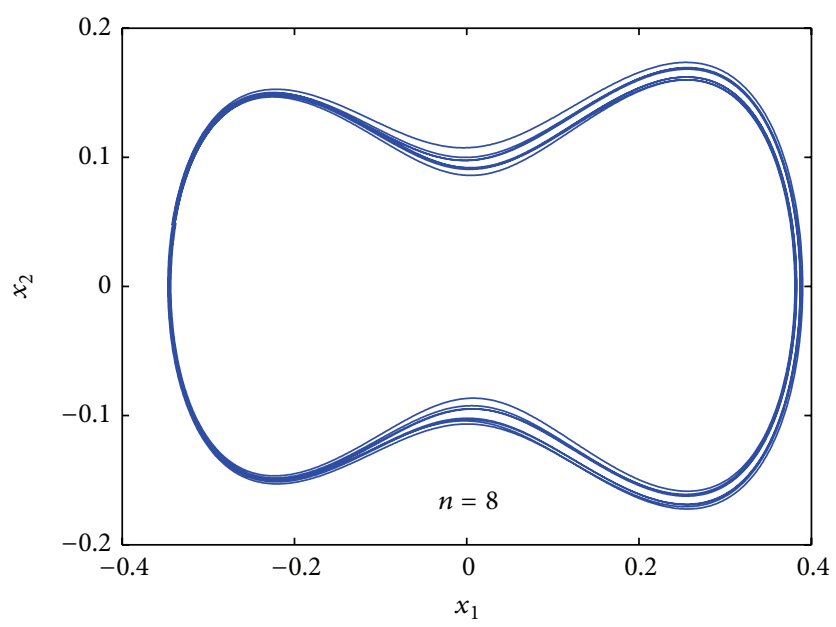

(f)

FIGURE 4: Unstable orbits found using close return method. The period of the orbit is identified with $n$. 


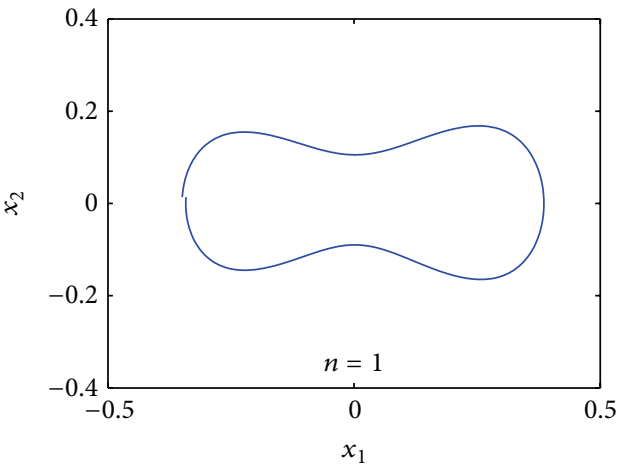

(a)

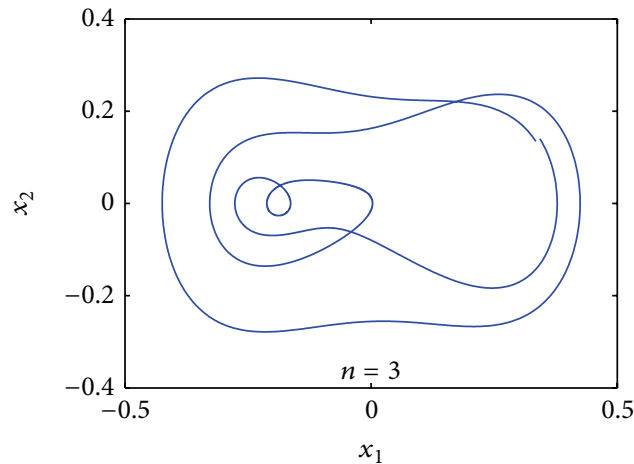

(c)

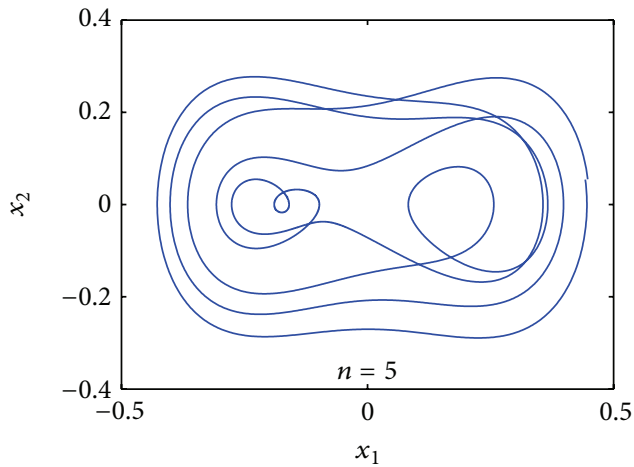

(e)

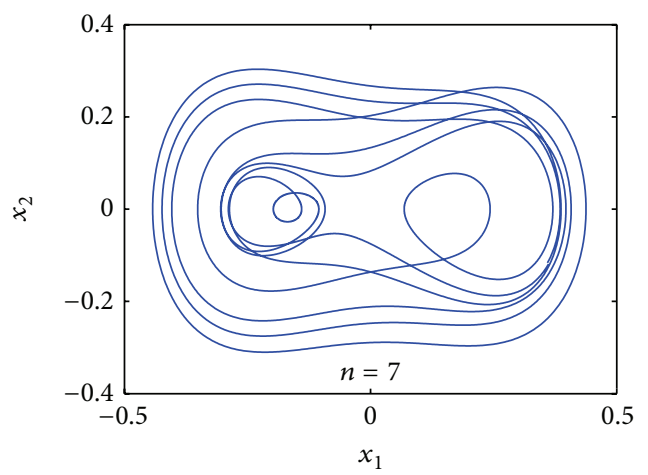

(g)

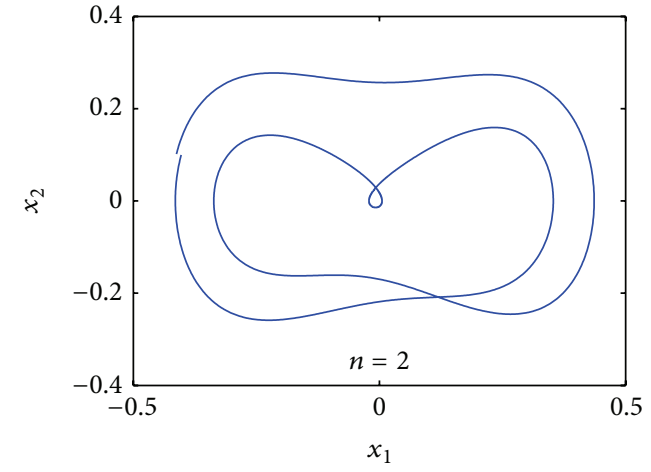

(b)

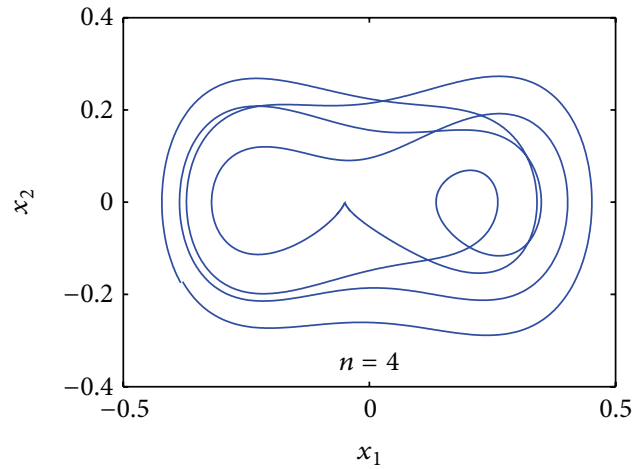

(d)

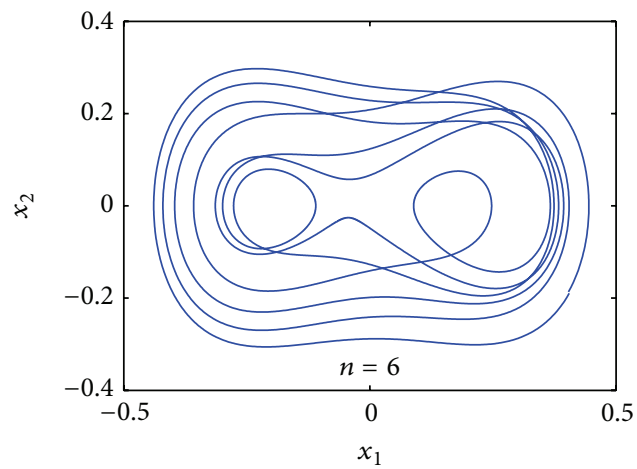

(f)

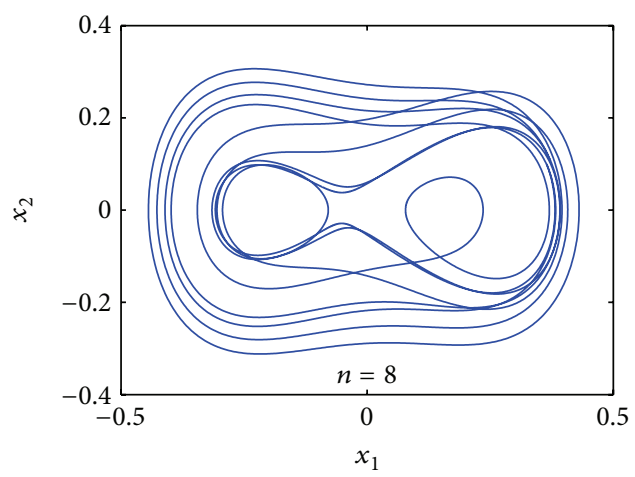

(h)

FIgURE 5: Unstable orbits found using close return method for a second set of data. The period of the orbit is $n$. 


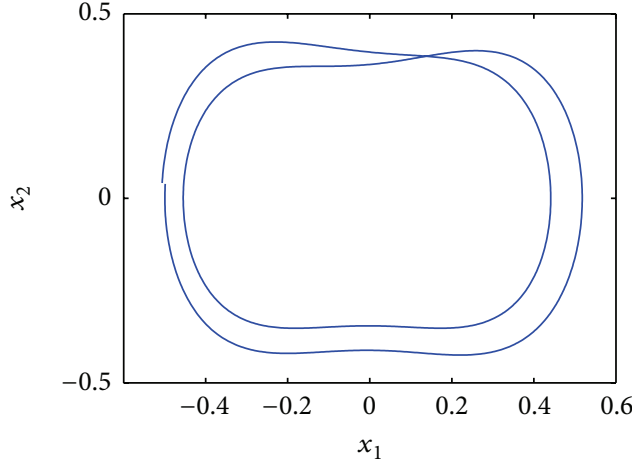

(a)

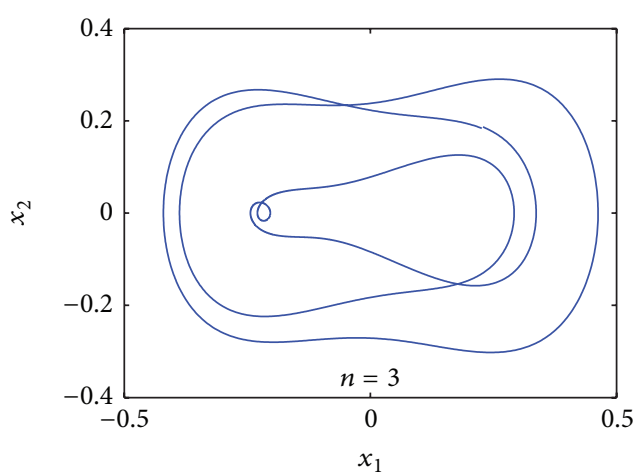

(c)

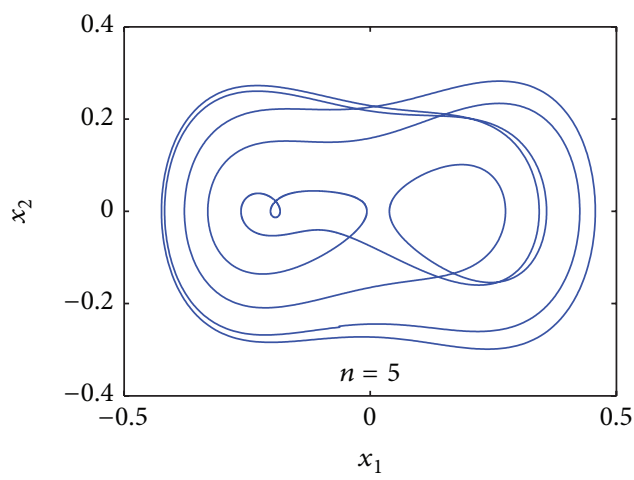

(e)

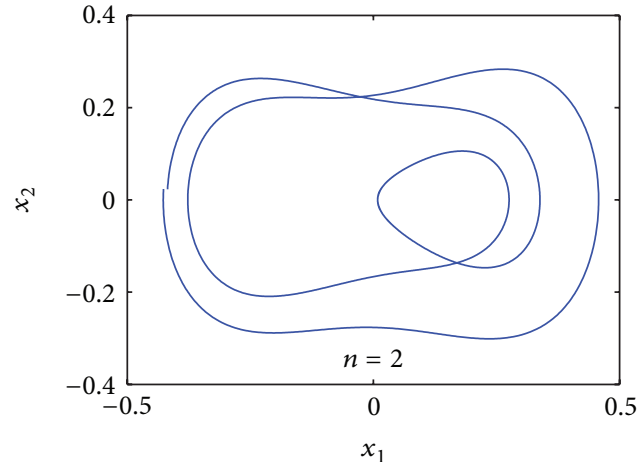

(b)

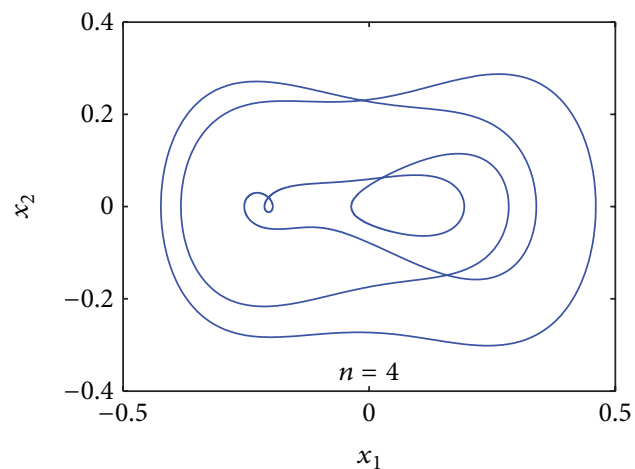

(d)

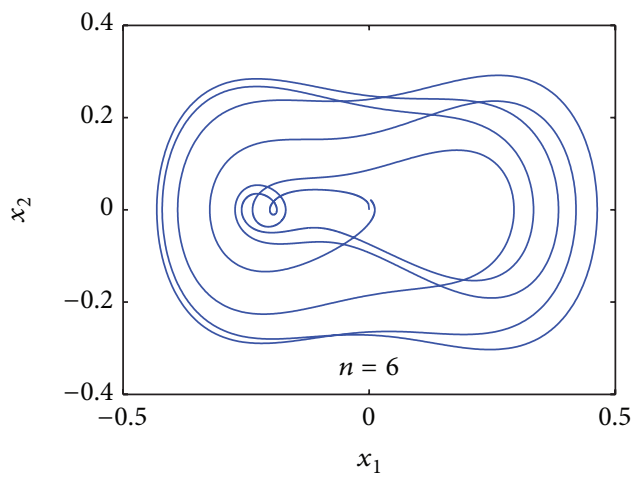

(f)

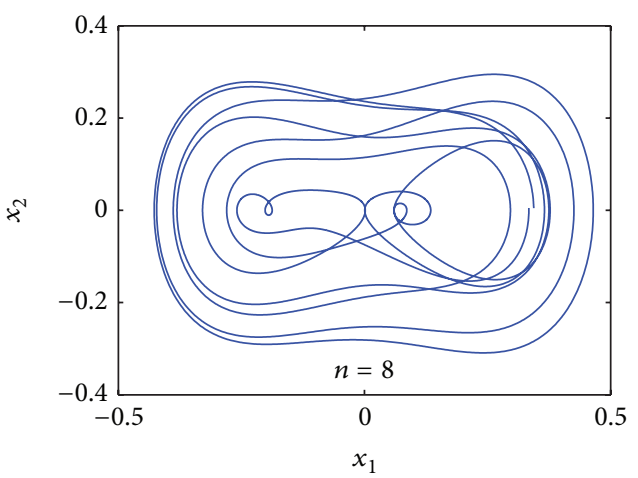

(g)

Figure 6: Unstable orbits found using close return method for a third set of data. $n$ identifies the period of the orbit. 


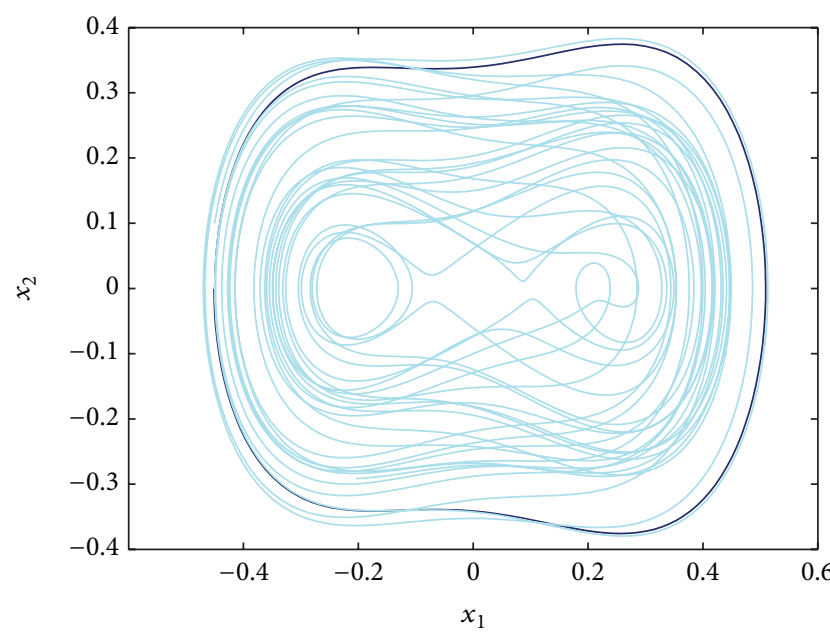

FIGURE 7: Unstable orbit surrounding the chaotic attractor.

where

$$
\begin{array}{cc}
a_{0}=0.0175400, & a_{1}=-0.4498000, \\
b_{1}=-0.000563, & a_{2}=0.0112600, \\
b_{2}=0.0008906, & a_{3}=-0.029140 \\
b_{3}=0.0000376, & a_{4}=0.0006330 \\
b_{4}=0.000231, & a_{5}=-0.001090 \\
\multicolumn{2}{c}{b_{5}=0.0000852}
\end{array}
$$

By taking the time derivative of (11), we obtain

$$
\begin{aligned}
\dot{y}_{r}= & a_{1}^{\prime} \cos \left(w_{1} t\right)+b_{1}^{\prime} \sin \left(w_{1} t\right)+a_{2}^{\prime} \cos \left(2 w_{1} t\right) \\
& +b_{2}^{\prime} \sin \left(2 w_{1} t\right)+a_{3}^{\prime} \cos \left(3 w_{1} t\right)+b_{3}^{\prime} \sin \left(3 w_{1} t\right) \\
& +a_{4}^{\prime} \cos \left(4 w_{1} t\right)+b_{4}^{\prime} \sin \left(4 w_{1} t\right)+a_{5}^{\prime} \cos \left(5 w_{1} t\right) \\
& +b_{5}^{\prime} \sin \left(5 w_{1} t\right),
\end{aligned}
$$

where

$$
\begin{array}{ll}
a_{1}^{\prime}=-0.00052, & b_{1}^{\prime}=0.4163, \\
a_{2}^{\prime}=0.0016, & b_{2}^{\prime}=-0.0208, \\
a_{3}^{\prime}=0.00010, & b_{3}^{\prime}=0.0809, \\
a_{4}^{\prime}=0.00085, & b_{4}^{\prime}=-0.0023, \\
a_{5}^{\prime}=0.00039, & b_{5}^{\prime}=0.00500 .
\end{array}
$$

The second derivative of $y_{r}$ is obtained by taking the derivative of (13).

In what follows, we use the following general assumption.

Assumption 1. The reference signal $y_{r}(t)$ and its first two derivatives are known and bounded. In addition, $y_{r}^{(p)}(t)$, $\forall p \geq 3$, is piecewise continuous.

\section{Output-Feedback Control of Chaotic MEMS}

The objective of the control is to stabilize the unstable orbit (11) of the chaotic uncertain system (6). This objective can be achieved by forcing the tracking error to tend to zero:

$$
\lim _{t \rightarrow \infty}\left[y(t)-y_{r}(t)\right]=0 .
$$

We use adaptive observer backstepping to deal with the parametric uncertainties in the model and also consider more realistic designs where only the output is available for measurement.

3.1. Adaptive Observer Backstepping Control. In order to apply a backstepping design procedure to system (6), we transform it into the parametric output-feedback form by using the following change of variables [20]:

$$
\begin{gathered}
x_{1}=\psi_{1}, \\
x_{2}=\psi_{2}+\mu \psi_{1} .
\end{gathered}
$$

System (6) becomes

$$
\begin{gathered}
\dot{x}_{1}=x_{2}-\theta_{1} x_{1}, \\
\dot{x}_{2}=-\theta_{2} x_{1}-\theta_{3} x_{1}^{3}+\theta_{4} f\left(x_{1}\right)+\beta(y) u, \\
y=x_{1},
\end{gathered}
$$

where $\theta=\left(\begin{array}{llll}\theta_{1} & \theta_{2} & \theta_{3} & \theta_{4}\end{array}\right)=\left(\begin{array}{llll}\mu & \sigma & \eta & \delta\end{array}\right)$ is a vector of uncertain parameters and

$$
\beta(y)=\frac{1}{(1-y)^{2}} .
$$

Equation (17) can be written in a more compact form

$$
\begin{gathered}
\dot{x}=A x+k y+b u+\varphi \theta, \\
y=c^{T} x,
\end{gathered}
$$

where $x=\left(\begin{array}{ll}x_{1} & x_{2}\end{array}\right)^{T}$ is the state vector, $y$ is the output, $u$ is the input, and $k=\left(\begin{array}{ll}k_{1} & k_{2}\end{array}\right)^{T}$ is a vector with $k_{1}, k_{2} \in \mathbb{Z}^{+}$, defined in such a way that the matrix $A$, given by

$$
A=\left[\begin{array}{ll}
-k_{1} & 1 \\
-k_{2} & 0
\end{array}\right]
$$

is Hurwitz, $b=\left(\begin{array}{ll}0 & \beta\end{array}\right)^{T}, \varphi$ is a nonlinear matrix defined by

$$
\begin{aligned}
\varphi & =\left[\begin{array}{cccc}
-x_{1} & 0 & 0 & 0 \\
0 & -x_{1} & -x_{1}^{3} & f\left(x_{1}\right)
\end{array}\right] \\
& =\left(\begin{array}{llll}
\varphi_{1} & \varphi_{2} & \varphi_{3} & \varphi_{4}
\end{array}\right),
\end{aligned}
$$

and $c=\left[\begin{array}{ll}1 & 0\end{array}\right]^{T}$.

We will reconstruct the state of the system through the use of the filters $\xi_{i}, i=0,1, \ldots, 4$. The state is reconstructed as

$$
x=\xi_{0}+\theta_{1} \xi_{1}+\theta_{2} \xi_{2}+\theta_{3} \xi_{3}+\theta_{4} \xi_{4}+\epsilon,
$$


where $\epsilon$ is the error between the state and its estimation. The virtual estimate of $x$ depends on the uncertain parameter $\theta$, but all we need in the design is the vector $\left(\begin{array}{lllll}\xi_{0} & \xi_{1} & \xi_{2} & \xi_{3} & \xi_{4}\end{array}\right)$. In order to guarantee that the error $\epsilon$ tends exponentially to be zero, we define the filters as

$$
\begin{gathered}
\dot{\xi}_{0}=\left[\begin{array}{ll}
-k_{1} & 1 \\
-k_{2} & 0
\end{array}\right]\left[\begin{array}{l}
\xi_{01} \\
\xi_{02}
\end{array}\right]+\left[\begin{array}{l}
k_{1} \\
k_{2}
\end{array}\right] y+\left[\begin{array}{l}
0 \\
\beta
\end{array}\right] u, \\
\dot{\xi}_{1}=\left[\begin{array}{ll}
-k_{1} & 1 \\
-k_{2} & 0
\end{array}\right]\left[\begin{array}{l}
\xi_{11} \\
\xi_{12}
\end{array}\right]+\left[\begin{array}{c}
-y \\
0
\end{array}\right], \\
\dot{\xi}_{2}=\left[\begin{array}{ll}
-k_{1} & 1 \\
-k_{2} & 0
\end{array}\right]\left[\begin{array}{l}
\xi_{21} \\
\xi_{22}
\end{array}\right]+\left[\begin{array}{c}
0 \\
-y
\end{array}\right], \\
\dot{\xi}_{3}=\left[\begin{array}{ll}
-k_{1} & 1 \\
-k_{2} & 0
\end{array}\right]\left[\begin{array}{l}
\xi_{31} \\
\xi_{32}
\end{array}\right]+\left[\begin{array}{c}
0 \\
-y^{3}
\end{array}\right], \\
\dot{\xi}_{4}=\left[\begin{array}{ll}
-k_{1} & 1 \\
-k_{2} & 0
\end{array}\right]\left[\begin{array}{l}
\xi_{41} \\
\xi_{42}
\end{array}\right]+\left[\begin{array}{c}
0 \\
f(y)
\end{array}\right]
\end{gathered}
$$

or in a compact way as

$$
\begin{gathered}
\dot{\xi}_{0}=A \xi_{0}+k y+b u, \\
\dot{\xi}_{j}=A \xi_{j}+\varphi_{j}(y), \quad j=1,2,3,4 .
\end{gathered}
$$

From (22) we can obtain the error dynamic equation

$$
\dot{\epsilon}=\dot{x}-\dot{\xi}_{0}-\theta_{1} \dot{\xi}_{1}-\theta_{2} \dot{\xi}_{2}-\theta_{3} \dot{\xi}_{3}-\theta_{4} \dot{\xi}_{4},
$$

and by using (19) and (23) we obtain

$$
\begin{aligned}
\dot{\epsilon}= & (A x+k y+b u+\varphi \theta)-\left(A \xi_{0}+k y+b u\right) \\
& -\theta_{1}\left(A \xi_{1}+\varphi_{1}\right)-\theta_{2}\left(A \xi_{2}+\varphi_{2}\right) \\
& -\theta_{3}\left(A \xi_{3}+\varphi_{3}\right)-\theta_{4}\left(A \xi_{4}+\varphi_{4}\right) \\
= & A\left(x-\xi_{0}-\theta_{1} \xi_{1}-\theta_{2} \xi_{2}-\theta_{3} \xi_{3}-\theta_{4} \xi_{4}\right) \\
= & A \epsilon .
\end{aligned}
$$

Since $A$ is Hurwitz, $\epsilon$ converges to zero exponentially.

The adaptive output-feedback controller is designed recursively applying backstepping as follows.

Step 1. We define the first error variable as the tracking error:

$$
z_{1}=y-y_{r}
$$

where $y_{r}$ is the reference signal which satisfies Assumption 1 .

Its dynamic is given by

$$
\begin{aligned}
\dot{z}_{1} & =\dot{y}-\dot{y}_{r}=\dot{x}_{1}-\dot{y}_{r} \\
& =x_{2}+\theta_{1}\left(-x_{1}\right)-\dot{y}_{r} ;
\end{aligned}
$$

since we cannot measure $x_{2}$, it cannot be used as a virtual control. We replace it with its virtual estimation and the corresponding error:

$$
x_{2}=\xi_{02}+\theta_{1} \xi_{12}+\theta_{2} \xi_{22}+\theta_{3} \xi_{32}+\theta_{4} \xi_{42}+\epsilon_{2} ;
$$

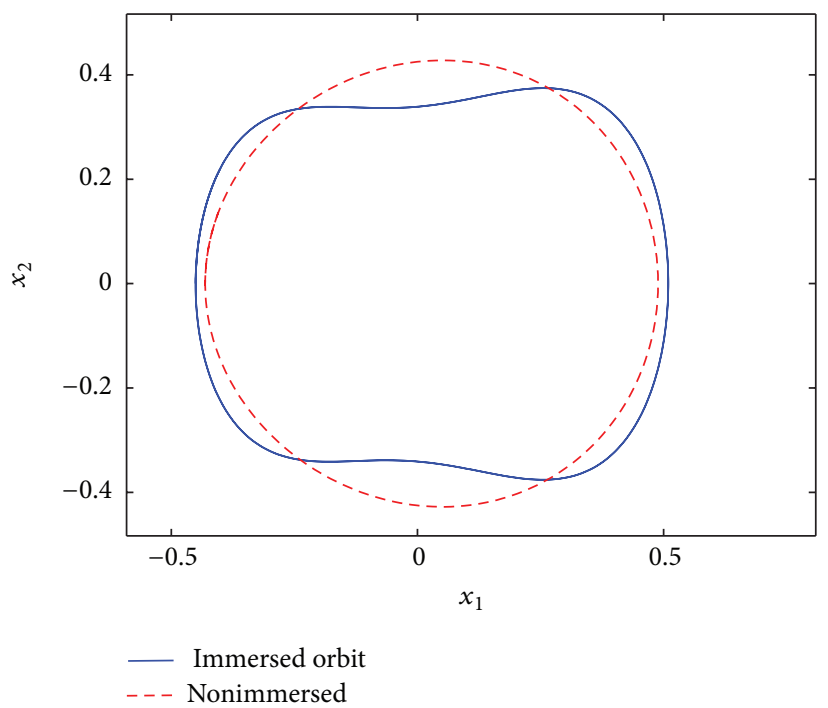

FIGURE 8: Unstable orbit immersed in the chaotic attractor and orbit not immersed.

hence (28) becomes

$$
\begin{aligned}
\dot{z}_{1}= & \xi_{02}+\theta_{1} \xi_{12}+\theta_{2} \xi_{22}+\theta_{3} \xi_{32}+\theta_{4} \xi_{42} \\
& +\theta_{1}\left(-x_{1}\right)-\dot{y}_{r}+\epsilon_{2} .
\end{aligned}
$$

By choosing $\xi_{02}$ as the virtual control and defining the second error variable as

$$
z_{2}=\xi_{02}-\alpha_{1}-\dot{y}_{r}
$$

(30) becomes

$$
\begin{aligned}
\dot{z}_{1}= & \left(z_{2}+\alpha_{1}+\dot{y}_{r}\right)+\theta_{1} \xi_{12}+\theta_{2} \xi_{22}+\theta_{3} \xi_{32} \\
& +\theta_{4} \xi_{42}+\theta_{1}\left(-x_{1}\right)-\dot{y}_{r}+\epsilon_{2} .
\end{aligned}
$$

Since $\theta$ is an uncertain vector, we must use its first estimated value $\vartheta_{1}=\left(\begin{array}{lllll}\vartheta_{11} & \vartheta_{12} & \vartheta_{13} & \vartheta_{14}\end{array}\right)^{T}$. By defining the first stabilizing function $\alpha_{1}$ as

$$
\alpha_{1}=-c_{1} z_{1}-d_{1} z_{1}-\vartheta_{1} \omega
$$

where $\omega=\left(\begin{array}{llll}\xi_{12}-x_{1} & \xi_{22} & \xi_{32} & \xi_{42}\end{array}\right)^{T}$, then (32) becomes

$$
\dot{z}_{1}=-c_{1} z_{1}+z_{2}-d_{1} z_{1}+\left(\theta-\vartheta_{1}\right) \omega+\epsilon_{2} .
$$

If we use the Lyapunov function candidate

$$
\begin{aligned}
V_{1}= & \frac{1}{2} z_{1}^{2}+\frac{1}{2 \gamma_{1}}\left(\theta-\vartheta_{1}\right)\left(\theta-\vartheta_{1}\right)^{T} \\
& +\frac{1}{d_{1}} \epsilon^{T} P_{0} \epsilon,
\end{aligned}
$$




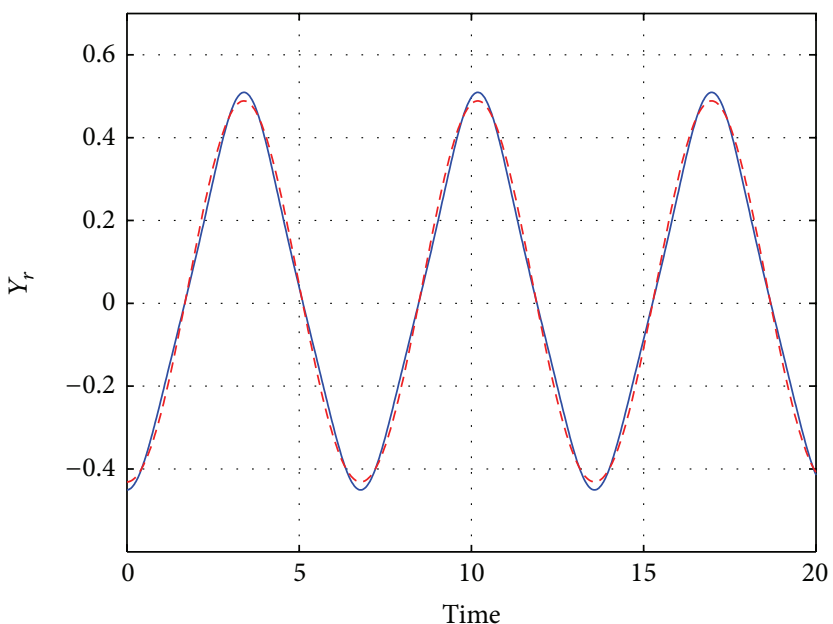

- Immersed

- - - Nonimmersed

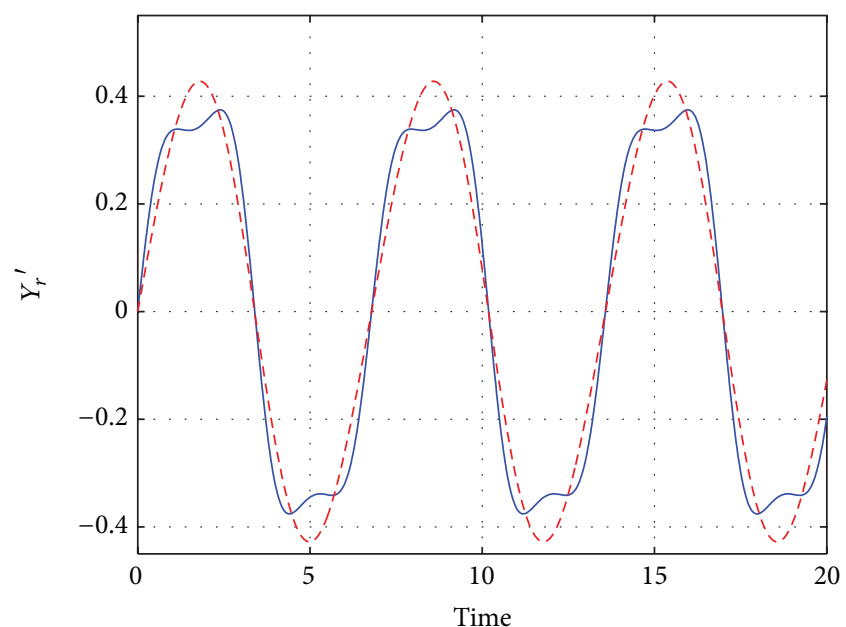

— Immersed

- - - Nonimmersed

(a)

(b)

FIGURE 9: Reference signal and its derivative: (a) $y_{r}$; (b) $\dot{y}_{r}$.

where $\gamma_{1}>0$ is the adaptation gain and $P_{0}=P_{0}^{T}>0$ satisfies $P_{0} A+A^{T} P_{0}=-I$, then

$$
\begin{aligned}
\dot{V}_{1}= & z_{1} \dot{z}_{1}-\frac{1}{\gamma} \dot{\vartheta}_{1}\left(\theta-\vartheta_{1}\right)^{T}-\frac{1}{d_{1}} \epsilon^{T} \epsilon \\
= & z_{1}\left(-c_{1} z_{1}+z_{2}-d_{1} z_{1}+\left(\theta-\vartheta_{1}\right) \omega+\epsilon_{2}\right) \\
& -\frac{1}{\gamma}\left(\theta-\vartheta_{1}\right) \dot{\vartheta}_{1}^{T}-\frac{1}{d_{1}} \epsilon^{T} \epsilon \\
= & -c_{1} z_{1}^{2}+z_{1} z_{2}-d_{1} z_{1}^{2}+\left(\theta-\vartheta_{1}\right)\left(z_{1} \omega-\frac{1}{\gamma} \dot{\vartheta}^{T}\right) \\
& +z_{1} \epsilon_{2}-\frac{1}{d_{1}} \epsilon^{T} \epsilon \\
= & -c_{1} z_{1}^{2}+z_{1} z_{2}-d_{1}\left(z_{1}-\frac{1}{2 d_{1}} \epsilon_{2}\right)^{2} \\
& +\frac{1}{4 d_{1}} \epsilon_{2}^{2}-\frac{1}{d_{1}} \epsilon^{T} \epsilon+\left(\theta-\vartheta_{1}\right)\left(z_{1} \omega-\frac{1}{\gamma} \dot{\vartheta}_{1}^{T}\right) \\
\leqslant & z_{1} z_{2}-c_{1} z_{1}^{2}+\left(\theta-\vartheta_{1}\right)\left(z_{1} \omega-\frac{1}{\gamma} \dot{\vartheta}_{1}^{T}\right) \\
& -\frac{3}{4 d_{1}} \epsilon^{T} \epsilon .
\end{aligned}
$$

It is possible to eliminate the term $\left(\theta-\vartheta_{1}\right)$ by the update law

$$
\dot{\vartheta}_{1}^{T}=\gamma z_{1} \omega .
$$

Step 2. The derivative of $z_{2}$ is

$$
\begin{aligned}
\dot{z}_{2}= & \dot{\xi}_{02}-\dot{\alpha}_{1}-\ddot{y}_{r} \\
= & \beta u+k_{2}\left(x_{1}-\xi_{01}\right)-\ddot{y}_{r}-\dot{\alpha}_{1} \\
= & \beta u+k_{2}\left(x_{1}-\xi_{01}\right)-\frac{\partial \alpha_{1}}{\partial y} \\
& \times\left(\xi_{02}+\theta_{1} \xi_{12}+\theta_{2} \xi_{22}+\theta_{3} \xi_{32}+\theta_{4} \xi_{42}+\epsilon_{2}+\theta_{1}\left(-x_{1}\right)\right) \\
& -\frac{\partial \alpha_{1}}{\partial \xi_{12}}\left(-k_{2} \xi_{11}\right)-\frac{\partial \alpha_{1}}{\partial \xi_{22}}\left(-k_{2} \xi_{21}-x_{1}\right) \\
& -\frac{\partial \alpha_{1}}{\partial \xi_{32}}\left(-k_{2} \xi_{31}-x_{1}^{3}\right)-\frac{\partial \alpha_{1}}{\partial \xi_{42}}\left(-k_{2} \xi_{41}+f_{1}\right) \\
& -\frac{\partial \alpha_{1}}{\partial y_{r}} \dot{y} r-\frac{\partial \alpha_{1}}{\partial \vartheta_{1}}\left(\gamma z_{1} \omega\right)-\ddot{y}_{r} .
\end{aligned}
$$

The control signal is chosen as

$$
\begin{aligned}
\beta u= & -k_{2}\left(x_{1}-\xi_{01}\right)+\frac{\partial \alpha_{1}}{\partial \xi_{12}}\left(-k_{2} \xi_{11}\right) \\
& +\frac{\partial \alpha_{1}}{\partial \xi_{22}}\left(-k_{2} \xi_{21}-x_{1}\right)+\frac{\partial \alpha_{1}}{\partial \xi_{32}}\left(-k_{2} \xi_{31}-x_{1}^{3}\right) \\
& +\frac{\partial \alpha_{1}}{\partial \xi_{42}}\left(-k_{2} \xi_{41}+f_{1}\right)+\frac{\partial \alpha_{1}}{\partial y_{r}} \dot{y}_{r}+\frac{\partial \alpha_{1}}{\partial \vartheta_{1}}\left(\gamma z_{1} \omega\right) \\
& +\ddot{y}_{r}+\frac{\partial \alpha_{1}}{\partial y}\left(\xi_{02}+\vartheta_{2} \omega\right)-d_{2}\left(\frac{\partial \alpha_{1}}{\partial y}\right)^{2} z_{2} \\
& -c_{2} z_{2}-z_{1}
\end{aligned}
$$




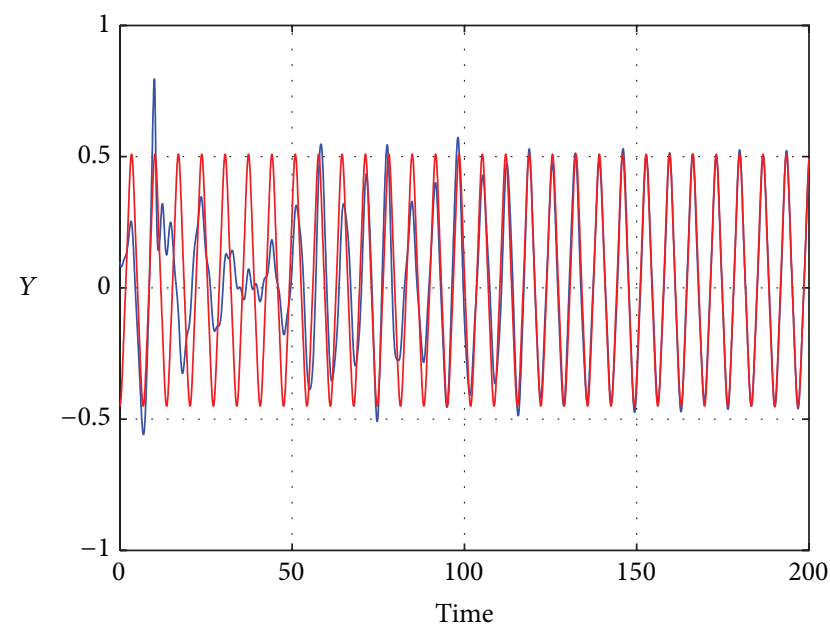

_ Reference orbit

— Stabilized orbit

(a)

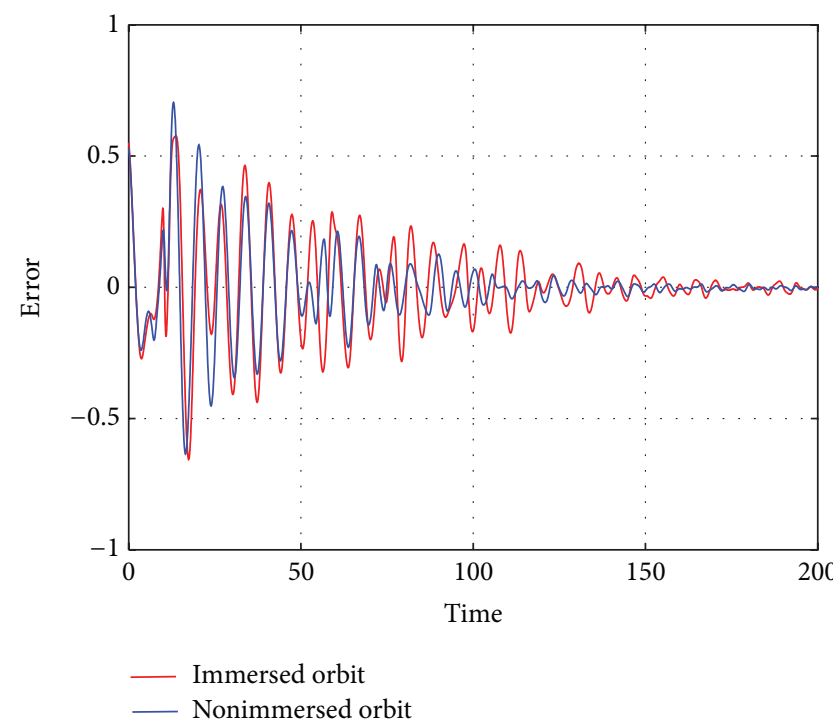

(c)

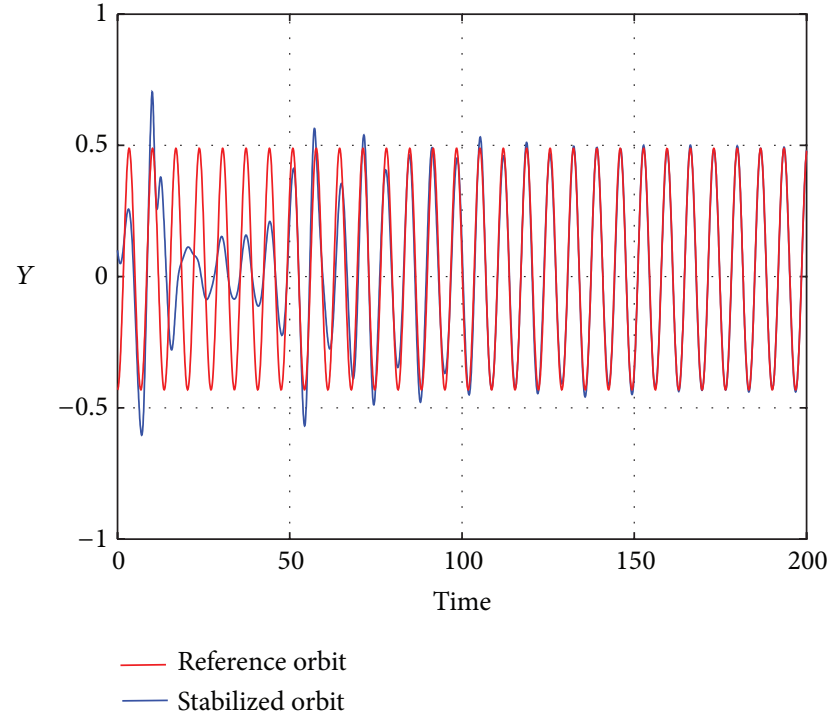

(b)

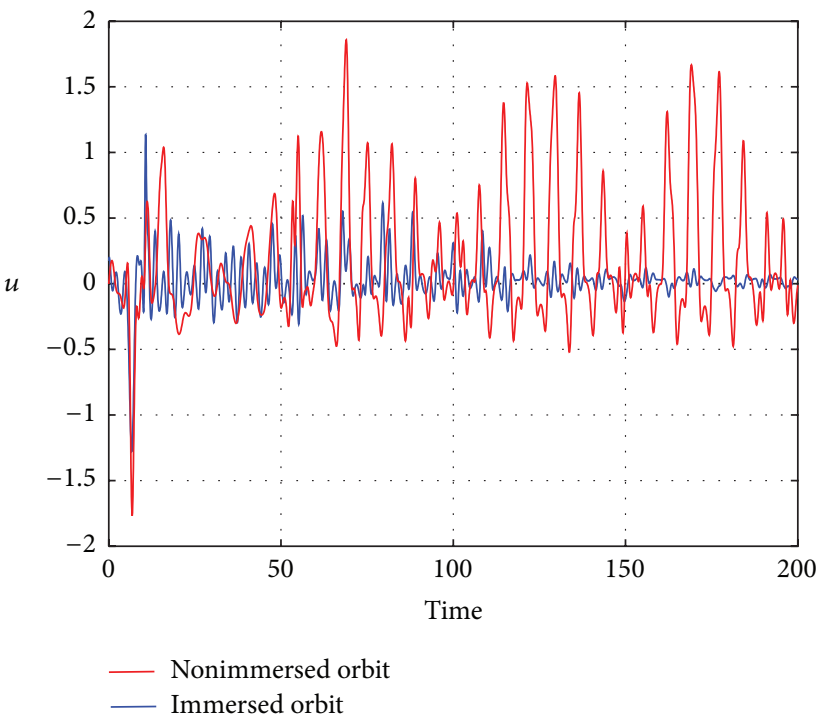

(d)

FIGURE 10: Simulation results for the controller: (a) output for the immersed orbit; (b) output for the nonimmersed orbit; (c) tracking errors; (d) control signals.

where $\vartheta_{2}=\left(\begin{array}{llll}\vartheta_{21} & \vartheta_{22} & \vartheta_{23} & \vartheta_{24}\end{array}\right)^{T}$ is a second estimate of $\vartheta$. The dynamic of the error $z_{2}$ is

$$
\begin{aligned}
\dot{z}_{2}= & -c_{2} z_{2}-z_{1}-d_{2}\left(\frac{\partial \alpha_{1}}{\partial y}\right)^{2} z_{2}-\frac{\partial \alpha_{1}}{\partial y} \epsilon_{2} \\
& -\frac{\partial \alpha_{1}}{\partial y}\left(\theta-\vartheta_{2}\right) \omega .
\end{aligned}
$$

Defining the Lyapunov function

$$
\begin{aligned}
V_{2}= & V_{1}+\frac{1}{2} z_{2}^{2}+\frac{1}{2 \gamma}\left(\theta-\vartheta_{2}\right)\left(\theta-\vartheta_{2}\right)^{T} \\
& +\frac{1}{d_{2}} \epsilon^{T} P_{0} \epsilon
\end{aligned}
$$

its derivative is computed as

$$
\begin{aligned}
\dot{V}_{2}= & \dot{V}_{1}+z_{2} \dot{z}_{2}-\frac{1}{\gamma} \dot{\vartheta}_{2}\left(\theta-\vartheta_{2}\right)^{T}-\frac{1}{d_{2}} \epsilon^{T} \epsilon \\
\leq & z_{1} z_{2}-c_{1} z_{1}^{2}-\frac{3}{4 d_{1}} \epsilon^{T} \epsilon-c_{2} z_{2}^{2}-z_{1} z_{2} \\
& -d_{2}\left(\frac{\partial \alpha_{1}}{\partial y}\right)^{2} z_{2}^{2}-\frac{\partial \alpha_{1}}{\partial y} \epsilon_{2} z_{2} \\
& -\left(\frac{\partial \alpha_{1}}{\partial y} \omega z_{2}+\frac{1}{\gamma} \dot{\vartheta}_{2}\right)\left(\theta-\vartheta_{2}\right)-\frac{1}{d_{2}} \epsilon^{T} \epsilon
\end{aligned}
$$




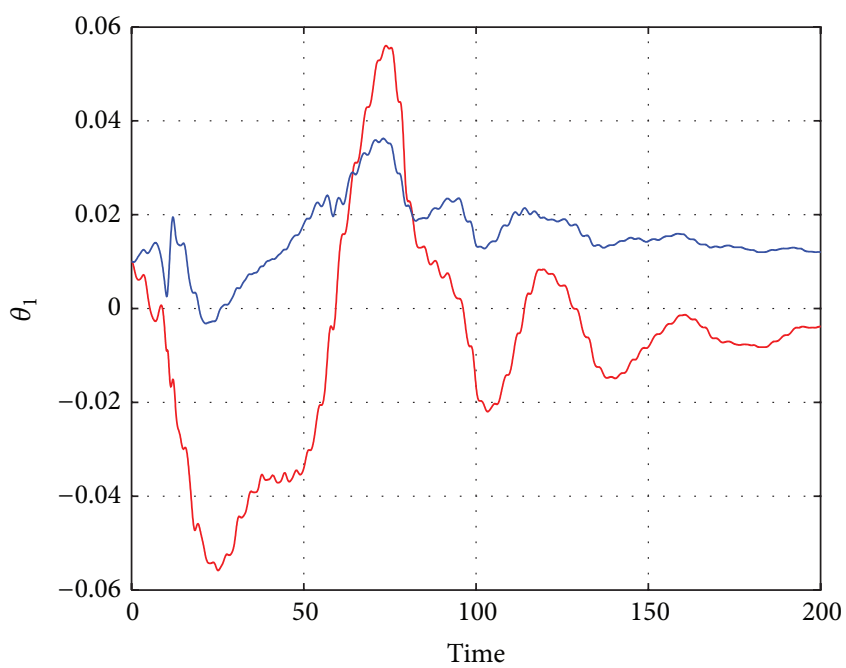

- First estimate - Second estimate

(a)

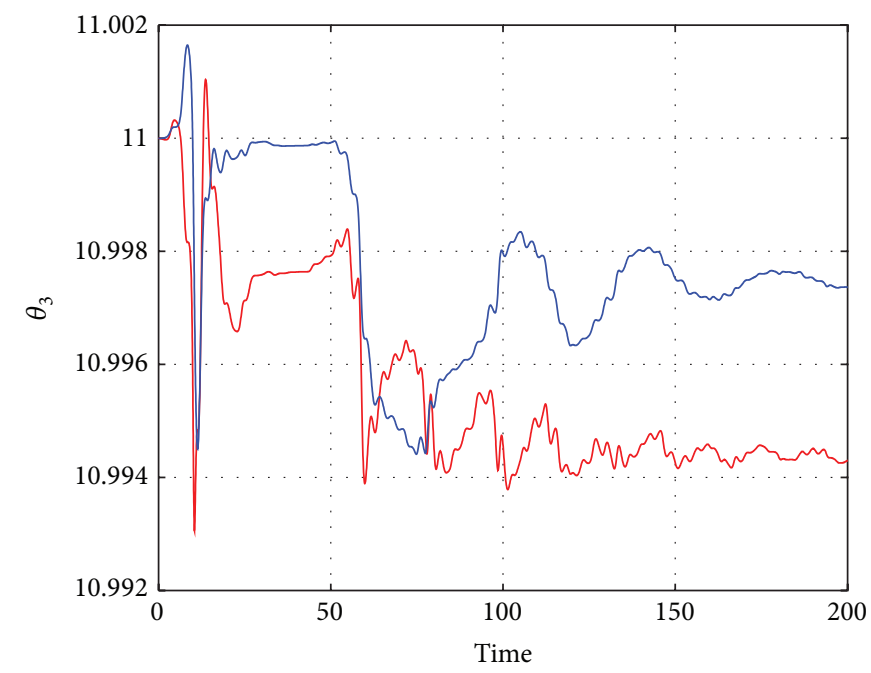

_ First estimate

- Second estimate

(c)

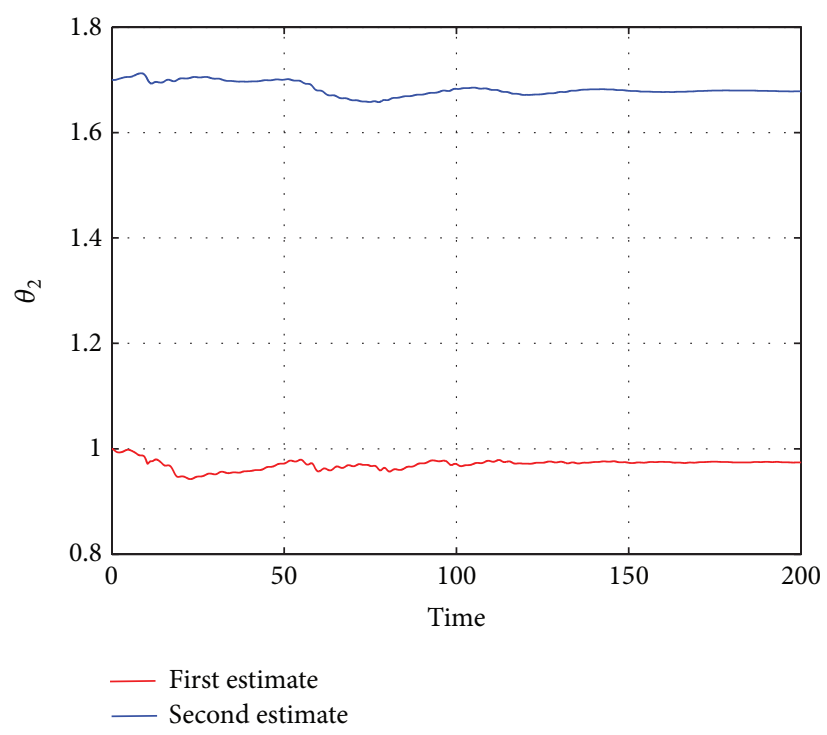

(b)

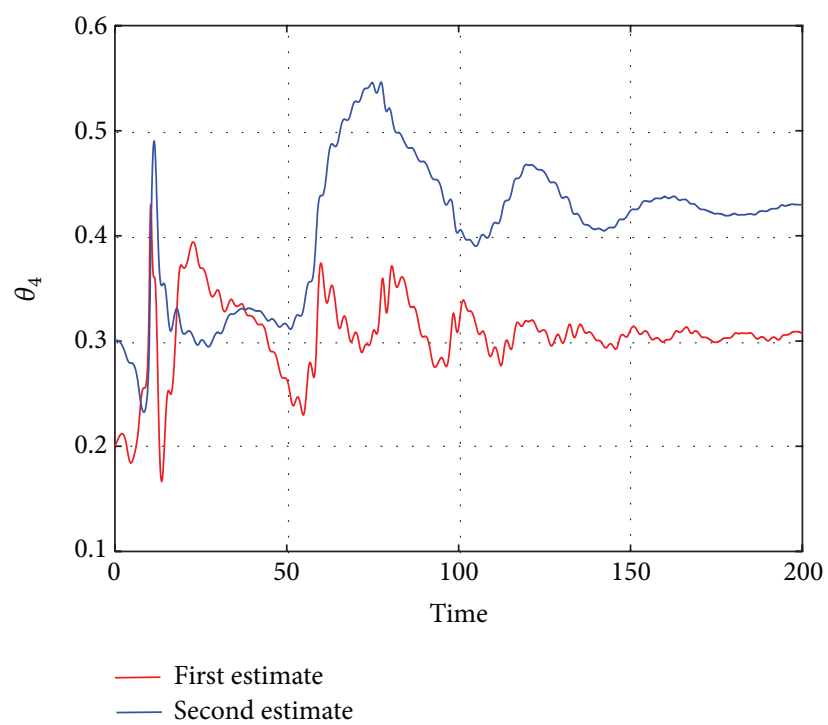

(d)

FIGURE 11: Estimated parameter $\vartheta$ for the immersed orbit: (a) $\vartheta_{11}$; (b) $\vartheta_{12}$; (c) $\vartheta_{13}$; (d) $\vartheta_{14}$.

$$
\begin{gathered}
\leq-c_{1} z_{1}^{2}-c_{2} z_{2}^{2}-\left(\frac{3}{4 d_{1}}+\frac{1}{d_{2}}\right) \epsilon^{T} \epsilon \\
-\left(\frac{\partial \alpha_{1}}{\partial y} \omega z_{2}+\frac{1}{\gamma} \dot{\vartheta}_{2}\right)\left(\theta-\vartheta_{2}\right) .
\end{gathered}
$$

To eliminate the term $\left(\theta-\vartheta_{2}\right)$ we use the update law

$$
\dot{\vartheta}_{2}=-\gamma \frac{\partial \alpha_{1}}{\partial y} \omega z_{2}
$$

and we finally obtain

$$
\dot{V}_{2} \leq-c_{1} z_{1}^{2}-c_{2} z_{2}^{2}-\left(\frac{3}{4 d_{1}}+\frac{1}{d_{2}}\right) \epsilon^{T} \epsilon .
$$

By applying the LaSalle-Yoshizawa theorem [20], we can conclude that $z_{1}, z_{2}, \vartheta_{1}$, and $\vartheta_{2}$ are bounded and $z \rightarrow 0$ as $t \rightarrow \infty$. Therefore, the output $y(t)$ tracks $y_{r}(t)$ globally and asymptotically.

\section{Simulation Results}

Numerical simulations have been carried out to validate the design made in Section 3. Two orbits are stabilized using the control strategy developed in the previous section with the aim of comparing the corresponding control effort.

First we will stabilize the unstable orbit (11) of the chaotic system (6), and then we stabilize the orbit:

$$
\begin{gathered}
y_{r 1}=a_{0}+1.15 a_{1} \cos \left(w_{1} t\right)+a_{2} \cos \left(2 w_{1} t\right), \\
\dot{y}_{r 1}=-1.15 a_{1} w_{1} \sin \left(w_{1} t\right)-2 w_{1} a_{2} \sin \left(2 w_{1} t\right),
\end{gathered}
$$




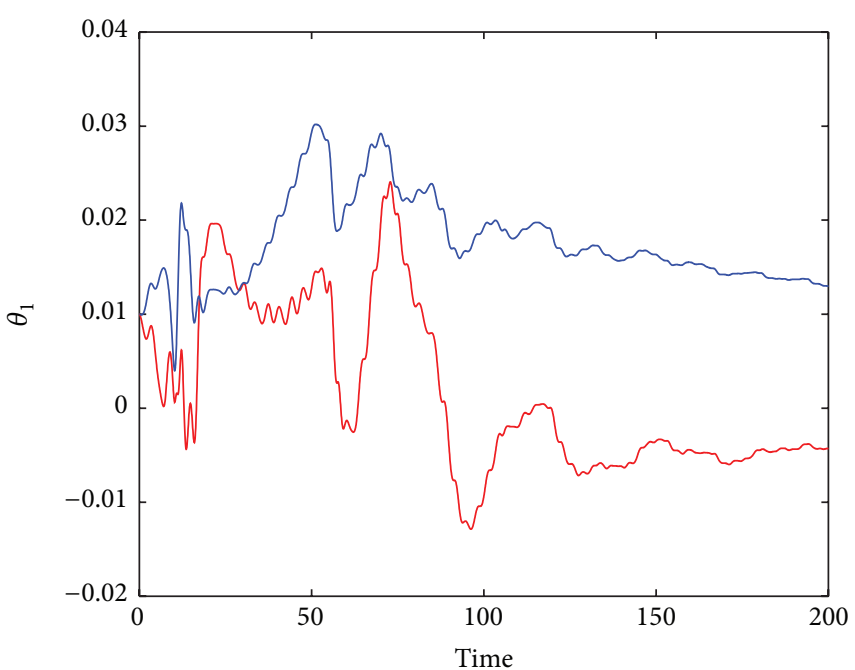

(a)

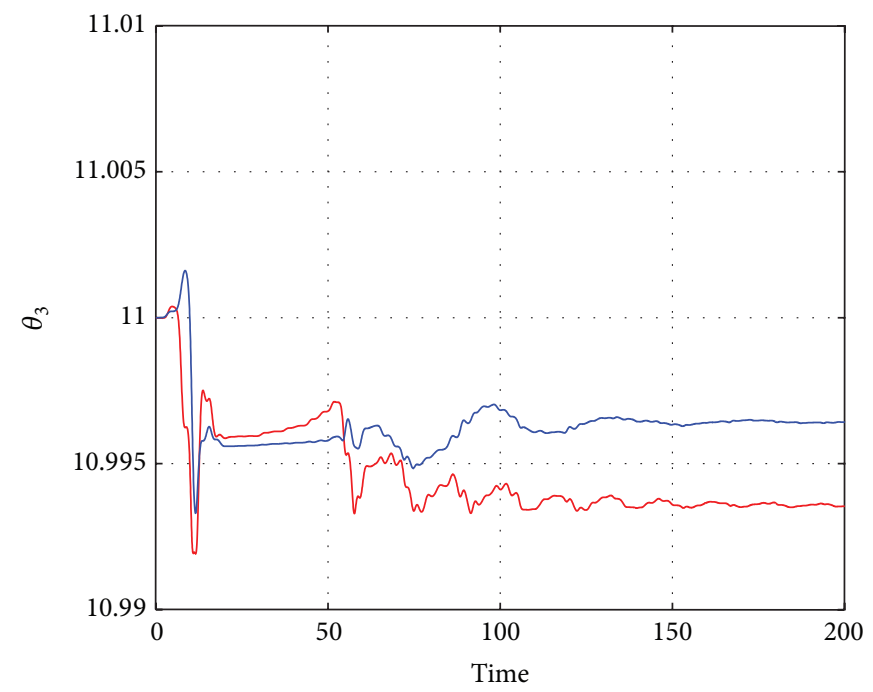

(c)

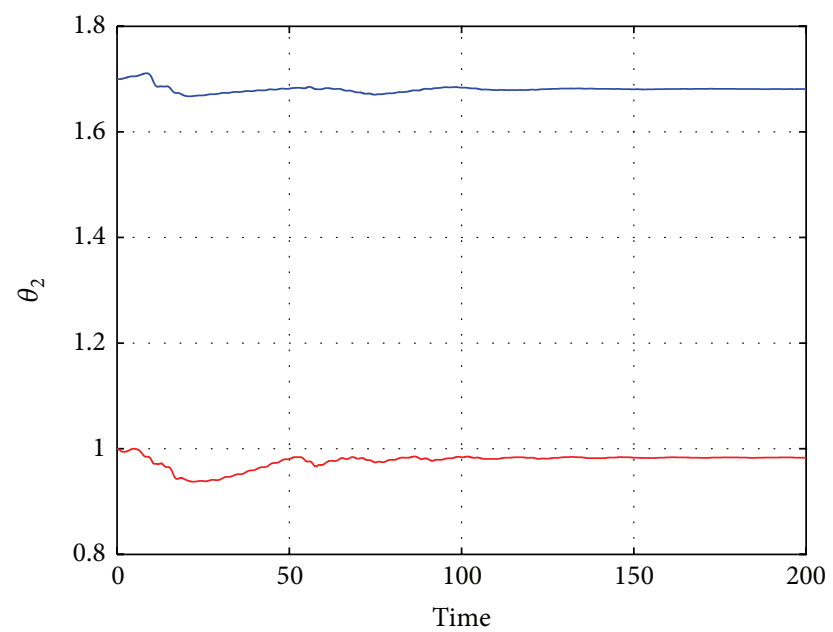

(b)

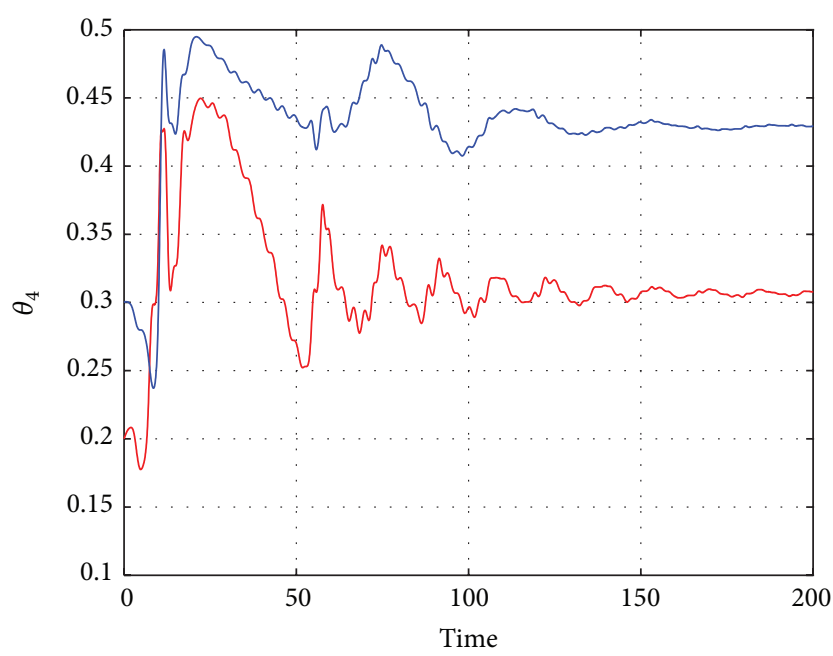

(d)

FIGURE 12: Estimated parameter $\vartheta$ for the nonimmersed orbit: (a) $\vartheta_{21}$; (b) $\vartheta_{22}$; (c) $\vartheta_{23}$; (d) $\vartheta_{24}$.

where

$$
a_{0}=0.01754, \quad a_{1}=-0.4, \quad a_{2}=0.01126 .
$$

These orbits have the same fundamental frequency $w_{1}$, and (45) is a perturbation of (11). The orbits are shown in Figure 8 and their states are compared in Figure 9. Although these two orbits are similar, one is immersed in the chaotic MEMS attractor while the other is not.

Figure 10(a) shows the output of the system when the orbit immersed in the chaotic attractor is stabilized by the algorithm developed in Section 3. In Figure 10(b) the output of the system is shown when the nonimmersed orbit is stabilized by using the same algorithm. The agreement between the curves and the effect of the controller can be seen.

The tracking errors are compared in Figure 10(c) from which we can observe a rapid decay as expected. A comparison between the control signals for stabilizing the two orbits is given in Figure 10(d). It clearly shows that, in steadystate, an affordable control effort is required for tracking the orbit immersed in the chaotic attractor, which is significantly smaller than that for the nonimmersed orbit.

Finally, the evolution of the estimates of the parameters given by the vector $\theta$ is shown in Figure 11 for the immersed orbit and in Figure 12 for the nonimmersed orbit. We can verify that the estimated values of the parameters are bounded as expected.

\section{Conclusions}

This work presented a solution for the enhancement of the oscillation amplitude of a MEMS resonator based on the chaotic properties of the system. In order to enhance the amplitude of the oscillation at a reduced control effort, a high amplitude unstable orbit immersed in the chaotic attractor was chosen as the reference trajectory. The method of close 
returns was used to compute the orbit, which was then approximated by a five-term truncated Fourier series. With the aim of stabilizing this orbit, a control law was designed by using output-feedback and considering parametric uncertainties in the model. It has been shown through adaptive observer backstepping that this control law allows the system output to globally and asymptotically track the desired orbits. Numerical simulations were carried out and the results show that the unstable orbits immersed in the chaotic attractor are stabilized with an affordable control effort compared to those required for stabilizing a nonimmersed orbit. This demonstrated the advantage of the proposed approach.

Finally, it is worth noting that the scheme developed in the present work is realistic in the sense that it is based on output-feedback and considers parametric uncertainties in the model. Variables as the speed of some components of the MEMS are very difficult to be measured, and a precise knowledge of the parameters of the system is hard due to the fabrication technology. In the approach proposed, the speed of the plates of the device was estimated by means of a virtual observer at the price of increasing the differential equations needed to construct the controller. Reducing the dynamic equations is beyond the scope of this paper and will be considered in a future work.

\section{Conflict of Interests}

The authors declare that there is no conflict of interests regarding the publication of this paper.

\section{References}

[1] H. S. Haghighi and A. H. D. Markazi, "Chaos prediction and control in MEMS resonators," Communications in Nonlinear Science and Numerical Simulation, vol. 15, no. 10, pp. 3091-3099, 2010.

[2] S. K. De and N. R. Aluru, "Complex oscillations and chaos in electrostatic microelectromechanical systems under superharmonic excitations," Physical Review Letters, vol. 94, no. 20, 2005.

[3] S. Liu, A. Davidson, and Q. Lin, "Simulation studies on nonlinear dynamics and chaos in a MEMS cantilever control system," Journal of Micromechanics and Microengineering, vol. 14, no. 7, pp. 1064-1073, 2004.

[4] A. C. J. Luo and F. Y. Wang, "Chaotic motion in a micro-electromechanical system with non-linearity from capacitors," Communications in Nonlinear Science and Numerical Simulation, vol. 7, no. 1-2, pp. 31-49, 2002.

[5] K. Park, Q. Chen, and Y.-C. Lai, "Energy enhancement and chaos control in microelectromechanical systems," Physical Review E, vol. 77, no. 2, Article ID 026210, 2008.

[6] G. Chen and X. Dong, "Ordering chaos of Chua's circuit," in Proceedings of the IEEE International Symposium on Circuits and Systems, pp. 2604-2607, May 1993.

[7] X. Dong and G. Chen, "Controlling chaotic continuous time systems via feedback," Nonlinear Analysis, vol. 69, p. 3409, 2008.

[8] A. Jimenez, E. N. Sanchez, J. P. Perez, and G. Chen, "Real-time chaos stabilization via inverse optimal control," in Proceedings of the IEEE International Symposium on Intelligent Control, ISIC '05 and the13th Mediterranean Conference on Control and
Automation (MED '05), pp. 1333-1336, Limassol, Cyprus, June 2005.

[9] Y. Deng, G. Sun, Y. Ye, F. Zhou, and K. Chen, "New chaos robust controller and its application," in Proceeding of the International Conference on Electric Information and Control Engineering (ICEICE '11), pp. 2387-2390, Wuhan, China, April 2011.

[10] S. S. Ge and C. Wang, "Adaptive control of uncertain Chua's circuits," IEEE Transactions on Circuits and Systems. I. Fundamental Theory and Applications, vol. 47, no. 9, pp. 1397-1402, 2000.

[11] A. Jimenez-Triana, G. Zhu, and L. Saydy, "Chaos synchronization of an electrostatic MEMS resonator in the presence of parametric uncertainties," in Proceedings of the American Control Conference (ACC '11), pp. 5115-5120, San Francisco, Calif, USA, July 2011.

[12] G. N. Nielson and G. Barbastathis, "Dynamic pull-in of parallelplate and torsional electrostatic MEMS actuators," Journal of Microelectromechanical Systems, vol. 15, no. 4, pp. 811-821, 2006.

[13] G. Zhu, "Electrostatic MEMS: modelling, control, and applications," in Advances in the Theory of Control, Signals and Systems with Physical Modeling, vol. 407 of Lecture Notes in Control and Information Sciences, pp. 113-123, Springer, Berlin, Germany, 2011.

[14] D. H. S. Maithripala, J. M. Berg, and W. P. Dayawansa, "Control of an electrostatic microelectromechanical system using static and dynamic output feedback," Journal of Dynamic Systems, Measurement and Control, vol. 127, no. 3, pp. 443-450, 2005.

[15] R. M. C. Mestrom, R. H. B. Fey, J. T. M. van Beek, K. L. Phan, and H. Nijmeijer, "Modelling the dynamics of a MEMS resonator: simulations and experiments," Sensors and Actuators A: Physical, vol. 142, no. 1, pp. 306-315, 2008.

[16] J. Awrejcewicz, K. Tomczak, and C. H. Lamarque, "Controlling systems with impacts," International Journal of Bifurcation and Chaos in Applied Sciences and Engineering, vol. 9, no. 3, pp. 547553, 1999.

[17] A. Wolf, J. B. Swift, H. L. Swinney, and J. A. Vastano, "Determining Lyapunov exponents from a time series," Physica D, vol. 16, no. 3, pp. 285-317, 1985.

[18] D. P. Lathrop and E. J. Kostelich, "Characterization of an experimental strange attractor by periodic orbits," Physical Review A, vol. 40, no. 7, pp. 4028-4031, 1989.

[19] J. Awrejcewicz, "Gradual and sudden transition to chaos in a sinusoidally driven nonlinear oscillator," Journal of the Physical Society of Japan, vol. 58, no. 12, pp. 4261-4264, 1989.

[20] M. Krstic, I. Kanellakopoulos, and P. V. Kokotovic, Nonlinear and Adaptive Control Design, John Wiley \& Sons, New York, NY, USA, 1995. 


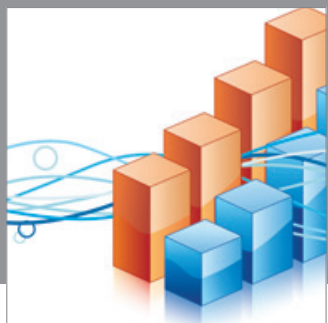

Advances in

Operations Research

mansans

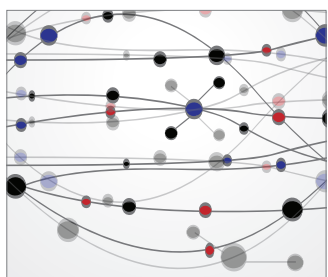

The Scientific World Journal
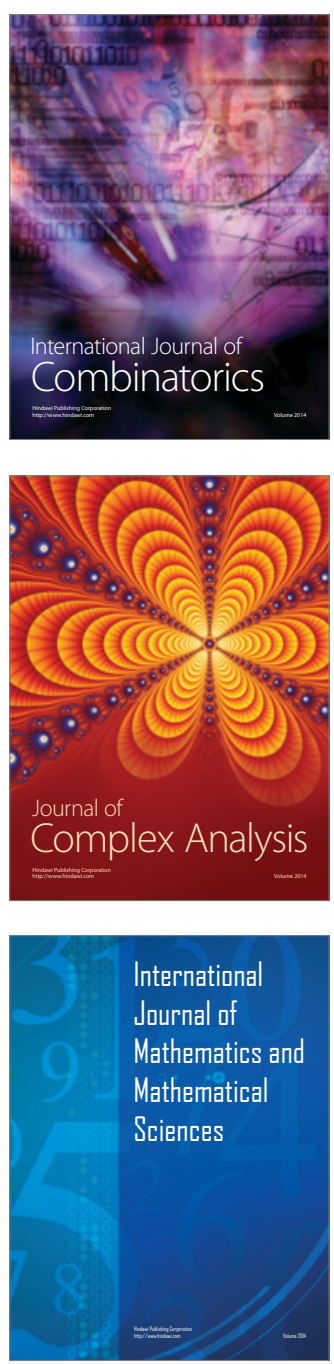
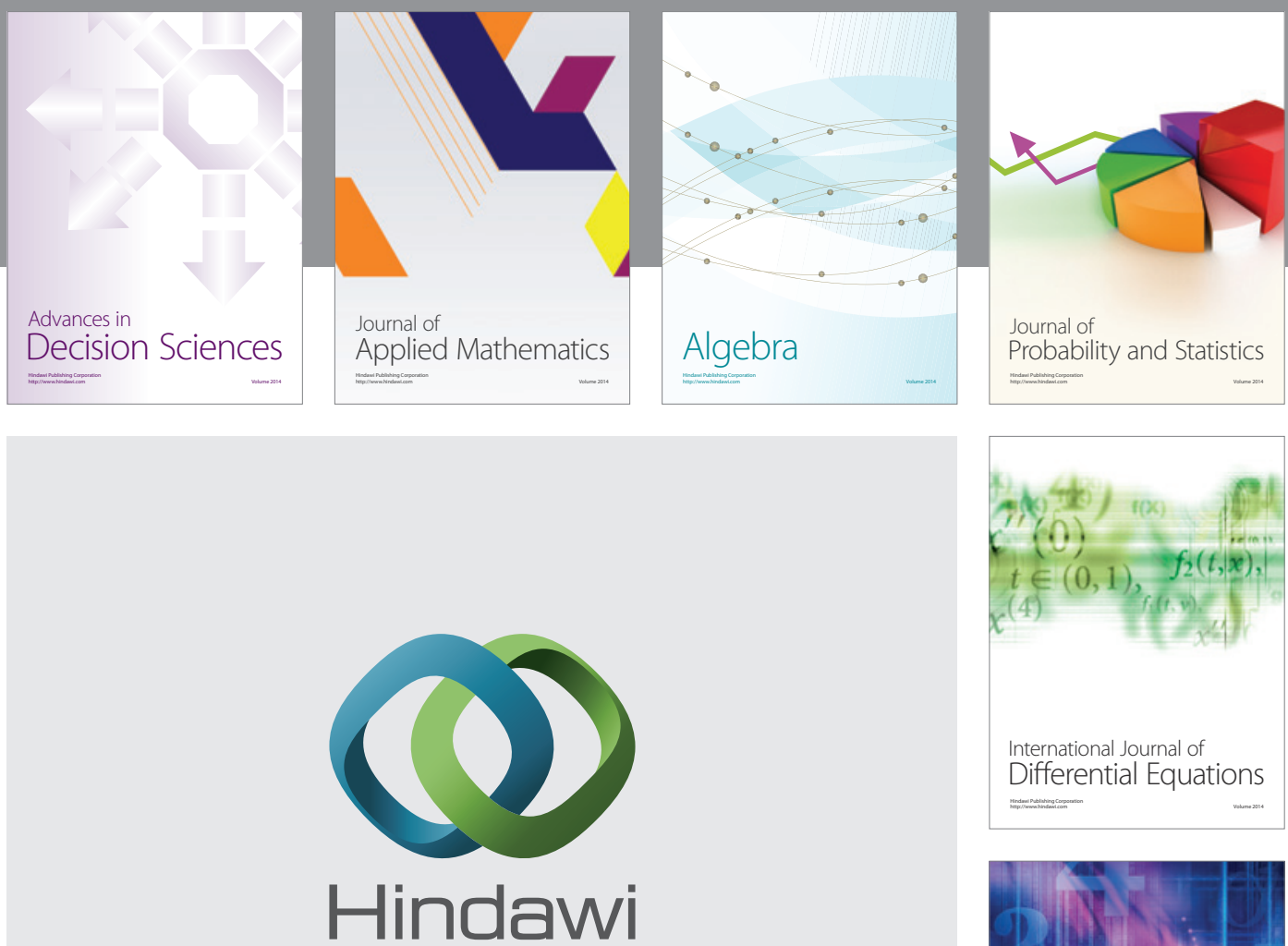

Submit your manuscripts at http://www.hindawi.com
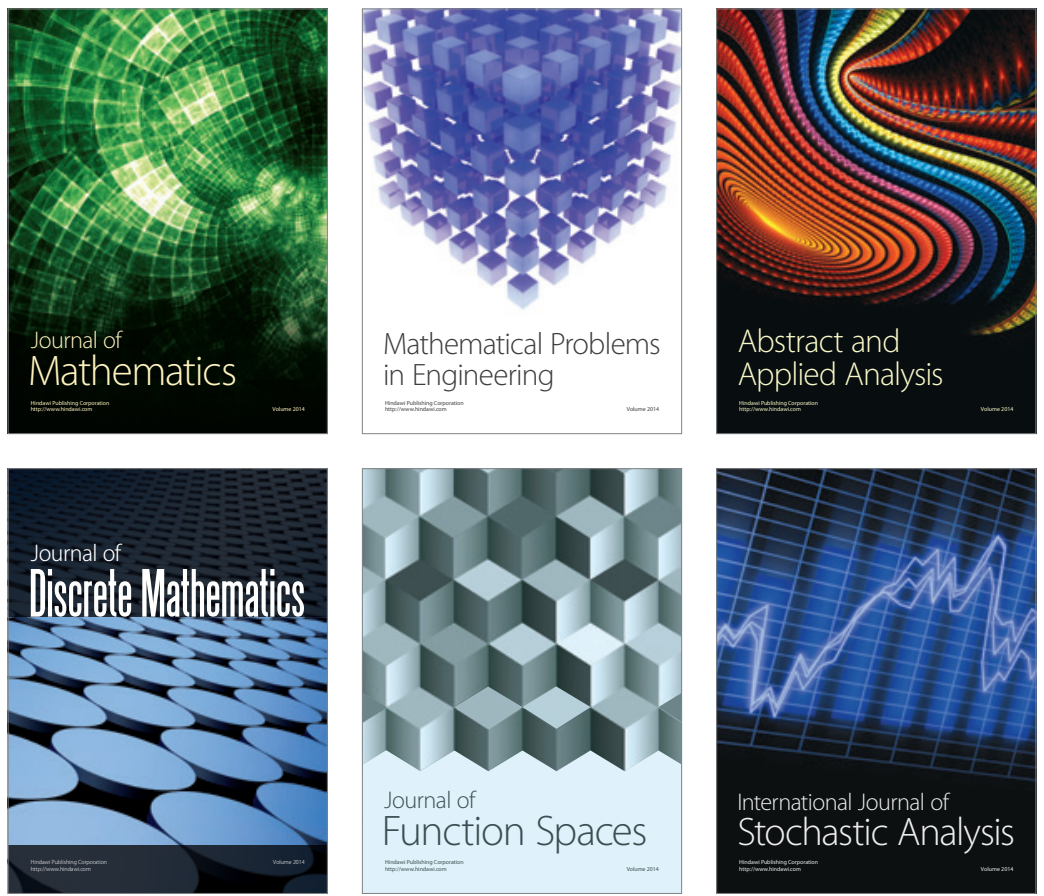

Journal of

Function Spaces

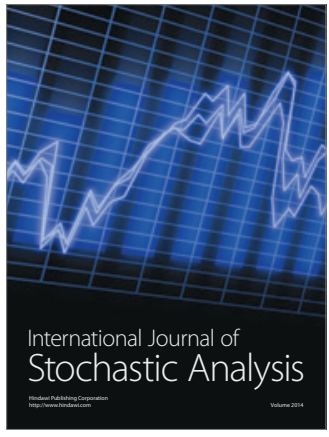

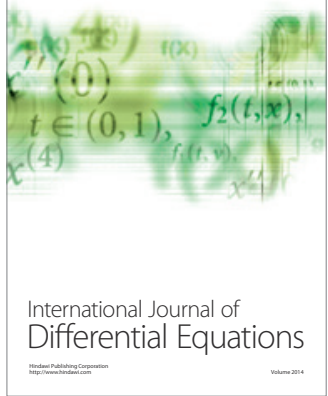
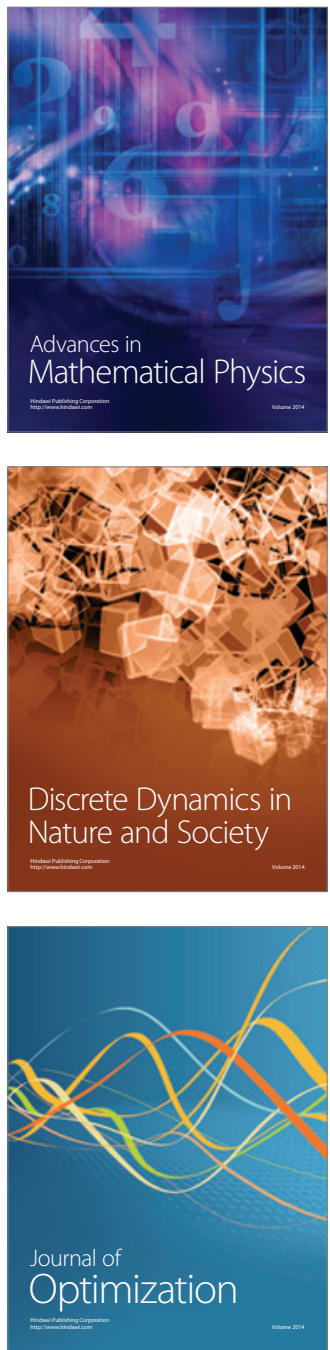